\title{
Nuclear-Spin Relaxation in Paramagnetic Solutions when the Electronic Zero-Field Splitting and Zeeman Interactions Are of Arbitrary Magnitude
}

\author{
ROBERT R. SHARP \\ Department of Chemistry, The University of Michigan, Ann Arbor, Michigan 48109 \\ Received February 3, 1992; revised March 13, 1992
}

\begin{abstract}
Expressions for the dipolar nuclear-spin relaxation rates in paramagnetic salt solutions have been derived under conditions where the electronic zero-field splitting (zfs) and Zeeman interactions are of arbitrary magnitude and when electron-spin relaxation is rapid compared to molecular reorientation. The theory is intended to provide continuity between the limiting analytical expressions previously derived for the Zeeman limit [SolomonBloembergen-Morgan (SBM) theory] and the zfs limit (R. Sharp, J. Chem. Phys. 93, 6921,1990 ). The more general solutions parallel the forms of both of these limiting theories in that they are comprised of sums of terms, each term consisting of a mean-square dipolar coupling energy times a spectral density function at one of the transition frequencies of the coupled I-S spin system. Geometric aspects of the problem are exhibited in simplest form in terms of spherical tensors, and the resulting expressions reduce in a straightforward manner to the Zeeman- and zfs-limit equations. As in the limiting theories, the electronspin relaxation time is treated as a parameter of the theory rather than calculated in detail from the time dependence of the electron-spin Hamiltonian. The theory has been applied to the analysis of magnetic field-dependent proton relaxation data of the ligand methyl protons in solutions of tris(acetylacetonato) $\mathrm{Mn}$ (III). The agreement with experiment is much superior to that found for SBM theory. $\odot 1992$ Academic Press, Inc.
\end{abstract}

The presence of paramagnetic transition metal ions in solutions and suspensions frequently causes pronounced perturbations of nuclear-spin relaxation times $\left(T_{1}\right.$ and $T_{2}$ ) in these systems. Measurements of the paramagnetic increment of nuclear-spin relaxation rates $\left[R_{1(2) p} \equiv\left(T_{1(2) p}\right)^{-1}\right]$ frequently provide a useful probe of the chemical and magnetic properties of the metal-ion binding site.

The physical interpretation of nuclear-spin relaxation in paramagnetic solutions was first developed by Solomon (1) and Bloembergen and Morgan (2, 3) (SBM theory). This theory, which expresses the paramagnetic relaxation increment in simple analytical expressions, is very convenient to apply and is still used almost universally in the practical interpretation of relaxation phenomena in paramagnetic solutions. The SBM equations have been generalized in several ways, for example, to include effects of anisotropy in the electronic $g$ factor and to incorporate multiple electron-spin relaxation times (4). The theory has a fundamental limitation however in that it is a limiting theory which assumes that the electron-spin Hamiltonian is the electronic Zeeman Hamiltonian $\mathscr{H}_{Z}$, i.e., it neglects effects of zero-field splittings except as a mechanism of electron-spin relaxation. Thus the theory is appropriate when the $\mathrm{zfs}$ is absent $(S$ 
$=\frac{1}{2}$ radicals or metal ions, e.g., $\mathrm{Cu}^{2+}$ ) or small [particularly ions possessing orbital singlet ground states, e.g., $\mathrm{Mn}$ (II), Gd(III)]. When the zfs is comparable to, or larger than, the Zeeman energy, the assumptions underlying SBM theory break down. In this situation, not only is the theory physically inappropriate, but no simple method exists for estimating the attendant error.

Recently, a parallel theory appropriate to the opposite limiting situation (the zfs limit), where the Zeeman energy is negligible compared to the zfs interaction, has been published $(5,6)$. In the zfs limit, the angular momentum of the electron spin is quantized along molecular axes rather than along the laboratory magnetic field. Appropriate to this physical situation, the motion of the electron spin was described in terms of tensor operators expressed in the molecular coordinate frame and the motion of the nuclear spin in terms of operators defined in the laboratory frame. Using this choice of coordinate systems, the time dependence of the electron-spin operators in the zfs limit can be expressed in a rather simple analytical form. The resulting theoretical expressions closely parallel the form of SBM theory and can be applied in practical situations with similar ease.

The objective of the present work was the development of theory which bridges the zfs and Zeeman limits and which maintains the basic mathematical form of the limiting theories, reducing to the limiting equations in a physically and geometrically transparent manner. The resulting theoretical formulation is valid for general $S$ and is reasonably simple to apply in practical situations.

Two rather serious problems are encountered in attempts to generalize the limiting theories, both associated with the calculation of the time correlation functions $\left\langle S_{ \pm}(t) S_{\mp}(0)\right\rangle$ of the electron-spin operators. The motion of the transverse components of $\mathbf{S}$ ( $\mathbf{S}$ is the spin vector of a spin with quantum number $S$ ) consists of a precessional component, the frequency of which is an eigenvalue of the spin system, and a stochastic portion reflecting effects of spin relaxation, i.e., transitions among the spin states. In the Zeeman and zfs limits, the precessional motion is stationary with a frequency which is an eigenvalue of a static Hamiltonian, either $\mathscr{H}_{\mathrm{Z}}$ or $\mathscr{H}_{\mathrm{ffs}}$. The limiting theories assume that stochastic relaxation processes are superimposed on this precessional motion and are exponential in form, characterized by one or more relaxation times $\tau_{\mathrm{S}}$. In the intermediate region, the electron-spin Hamiltonian depends on the relative orientations of the laboratory and molecular coordinate systems. In this situation, the frequency of the precessional motion is not stationary but rather is a stochastic function of molecular Brownian motion. Relaxation processes, due either to vibrational damping processes or to stochastic reoricntational motions, cause additional time dependence in the spin operators. In the most general strong-coupling situation, calculation of the time correlation functions of the components of the electron spin is very complex. Methodologies based on the stochastic Liouville equation have been developed and applied to physical situations outside the limiting regimes $(7-16)$. These treatments are not very simple to apply in practical situations, however, and they lack the transparent physical and geometrical forms of the limiting theories.

The present treatment follows an alternative approach and assumes that molecular motion is slow relative to the time scale of electron-spin relaxation. In this case the precessional part of the electron-spin Hamiltonian can be treated as a static function of molecular orientation. This assumption is most clearly applicable for transition 
metal ions complexed to high-molecular-weight species, for example, to metalloenzymes, to metal-nucleic acid complexes, or to metals bound to membranes. The slowmotion assumption $\left(\tau_{\mathrm{S}} \ll \tau_{\mathrm{R}}\right)$ is by no means restricted to such systems, however. For metal ions with substantial zero-field splittings, the electron-spin relaxation time $\tau_{\mathrm{S}}$ tends to be quite short. Physically, this is due to the fact that electron-spin relaxation results from stochastic modulation of the electron-spin Hamiltonian, which results from rotational and vibrational modulation of the $\mathrm{zfs}$ interaction or from rotational modulation of the Zeeman interaction. A large static zfs is normally associated with large derivatives of the zfs energy with respect to the vibrational and reorientational variables of molecular motion. Thus ions with large zero-field splittings normally possess very short clectron-spin relaxation times, frequently shorter than the reorientational correlation time $\tau_{R}$ of the metal-ion coordination sphere, even in lowmolecular-weight complexes.

As a practical example, $\mathrm{Mn}$ (III) is typically associated with fairly large zero-field splittings [measured values are in the range $\left.1-4 \mathrm{~cm}^{-1}(17-20)\right]$. The values of $\tau_{\mathrm{S}}$ of $\mathrm{Mn}(\mathrm{III})$ in the two solution systems in which this quantity has been measured $\left[\mathrm{Mn}\left(\mathrm{H}_{2} \mathrm{O}\right)_{6}^{3+}(21)\right.$ and tris(acetylacetonato $\left.) \mathrm{Mn}(\mathrm{III})(5)\right]$ are very short $(<10 \mathrm{ps})$ and approximately field-independent. In comparison, the reorientational correlation times $\tau_{\mathrm{R}}$ can be estimated, using Debye's theory, to be several times longer. Thus an approximation of slow molecular motion is reasonable even though the metal complexes are low-molecular-weight species. Transition metal ions which are likely to show similar behavior include (among others) high-spin $\mathrm{Fe}$ (II), $\mathrm{Ni}$ (II), and $\mathrm{Co}$ (II), as well as most of the common oxidation states of the lanthanide ions, with the exception of Gd(II) and Dy (III).

The theory developed here has been applied to the interpretation of magnetic fielddependent $T_{1}$ data (22) of methyl protons on the acetylacetonato (AcAc) ligand in solutions containing $\mathrm{Mn}(\mathrm{III})(\mathrm{AcAc})_{3}$ measured over a range of magnetic field strengths of $0.2-6.5 \mathrm{~T}$. At the lower end of this range, $\mathscr{H}_{\mathrm{Z}} \ll \mathscr{H}_{\mathrm{zfs}}$, and $R_{1 p}$ data should conform to zfs-limit theory, while at the upper end, the $\mathrm{zfs}$ and Zeeman energies are of comparable magnitude. An attractive feature of the $\mathrm{Mn}(\mathrm{III})(\mathrm{AcAc})_{3}$ system is that the electron-spin relaxation time $\tau_{\mathrm{S}}$ of $\mathrm{Mn}$ (III) has been measured independently through an analysis of intermolecular proton relaxation data of the solvent protons in acetone solutions using the zfs-limit theory (5). The measured value is very short, $\tau_{\mathrm{S}}=8 \pm 2$ ps, indicating that the slow-motion approximation is appropriate. For these reasons, $\mathrm{Mn}(\mathrm{III})(\mathrm{AcAc})_{3}$ provides an interesting system for application of the present theory.

\section{THEORETICAL}

Magnetic dipole Hamiltonian. The nuclear $T_{1 p}^{-1}$ and $T_{2 p}^{-1}$ arise physically from fluctuations in the dipolar and scalar parts of the electron-nuclear hyperfine coupling. For the magnetic dipole interaction, the coupling Hamiltonian can be written (in S.I. units)

$$
\mathscr{H}_{\mathrm{d}-\mathrm{d}}=\frac{\kappa}{r^{3}} \sum_{q} 3^{-1 / 2}(-1)^{1-q} I_{q}^{(1)} F_{-q}^{(1)}
$$


with

$$
\begin{gathered}
I_{ \pm 1}^{(1)}=\mp 2^{-1 / 2} I_{ \pm} \\
I_{0}^{(1)}=I_{z} \\
\kappa=-30^{1 / 2} \gamma_{\mathrm{I}} g \beta_{0}\left(\frac{\mu_{0}}{4 \pi}\right),
\end{gathered}
$$

where $\gamma_{\mathrm{I}}$ is the nuclear gyromagnetic ratio, $g$ is the electron $g$ factor, $\beta_{0}$ is the Bohr magneton, and $\mu_{0}$ is the magnetic permeability of free space. The spherical tensor $F^{(1)}$ describes the magnetic dipole coupling of the nuclear spin I to the thermal lattice. $F^{(1)}$ is a function of the variables of the electron-spin variables and of the polar spatial variables $(\vartheta, \phi)$ which specify the orientation of the I-S vector (of length $r$ ) in the laboratory coordinate frame. The components of $F^{(1)}$ can be constructed as the tensor product

$$
\mathbf{F}^{(1)}=\left\{\mathbf{S}^{(1)} \otimes \mathbf{C}^{(2)}\right\}^{(1)}
$$

where

$$
\begin{aligned}
& S_{ \pm 1}^{(1)}=\mp 2^{-1 / 2} S_{ \pm}, \\
& S_{0}^{(1)}=S_{z},
\end{aligned}
$$

and the $C_{m}^{(2)}$ are Racah's normalized spherical harmonics $(23,24)$,

$$
C_{m}^{(j)}(\vartheta, \phi)=\left(\frac{4 \pi}{5}\right)^{1 / 2} Y_{m}^{(j)}(\vartheta, \phi),
$$

where the $Y_{m}^{(2)}(\vartheta, \phi)$ are the usual spherical harmonics. Racah's spherical harmonics are very convenient to use in problems involving coordinate transformations since they are special forms of the Wigner rotation-matrix elements $D_{q, q}^{(j)},(\alpha, \beta, \gamma)$ :

$$
C_{q}^{(j)}(\vartheta, \phi)=(-1)^{q} \mathscr{D}_{q, 0}^{(j)}(0, \vartheta, \phi)=\mathcal{D}_{0, q}^{(j)}(\phi, \vartheta, 0) .
$$

Thus they are normalized to $(2 j+1)^{-1}$ over the space of the Euler angles, and their binary products, $C_{q}^{(j)}(\vartheta, \phi) C_{q^{\prime}}^{\left(j^{\prime}\right)},(\vartheta, \phi)$, can be contracted using the Clebsch-Gordon formula (a property that is employed extensively below). Specific components of $F^{(1)}$ can be constructed using the $3-j$ symbols

$$
F_{m}^{(1)}=\sum_{p=-1}^{1} \sum_{q=-2}^{2}(-1)^{1-q 3^{1 / 2}}\left[\begin{array}{ccc}
1 & 2 & 1 \\
p & q & m
\end{array}\right] S_{p}^{(1)} C_{q}^{(2)}(\vartheta, \phi) .
$$

This sum is restricted by the fact that the $3-j$ symbol vanishes unless $m=-(p+$ $q),|p| \leqslant 1,|q| \leqslant 2$, and $|m| \leqslant 1$, giving

$$
F_{m}^{(1)}=\sum_{p=-1}^{1} c_{m, p} S_{p}^{(1)} C_{m-p}^{(2)}(\vartheta, \phi)
$$


The coefficients $c_{m, p}$ can be evaluated using tabulated values of the $3-j$ symbols (24):

$$
\begin{aligned}
c_{+1,+1} & =c_{-1,-1}=10^{-1 / 2} \\
c_{+1,0} & =c_{-1,0}=-\left(\frac{3}{10}\right)^{1 / 2} \\
c_{0,+1} & =c_{0,-1}=\left(\frac{3}{10}\right)^{1 / 2} \\
c_{+1,-1} & =c_{-1,+1}=\left(\frac{3}{5}\right)^{1 / 2} \\
c_{0,0} & =-\left(\frac{2}{5}\right)^{1 / 2}
\end{aligned}
$$

From the density-matrix theory of nuclear-spin relaxation (25), the paramagnetic dipolar part of the $T_{1}$ and $T_{2}$ nuclear-spin relaxation rates is

$$
\begin{aligned}
&\left(T_{1 p}\right)_{\mathrm{dip}}^{-1}=(-1)\left(\frac{3^{-1 / 2} \kappa}{r^{3}}\right)^{2} \\
& \times\left\{\int_{0}^{\infty}\left\langle F_{+1}^{(1)}(t) F_{-1}^{(1)}(0)\right\rangle e^{-i \omega_{1} t}+\left\langle F_{-1}^{(1)}(t) F_{+1}^{(1)}(0)\right\rangle e^{i \omega_{1} t} d t\right\}_{\mathrm{sp} \mathrm{av}}, \\
&\left(T_{2 p}\right)_{\mathrm{dip}}^{-1}=2^{-1}\left(T_{1 p}\right)_{\mathrm{dip}}^{-1}+\left(\frac{3^{-1 / 2} \kappa}{r^{3}}\right)^{2}\left\{\int_{0}^{\infty}\left\langle F_{0}^{(1)}(t) F_{0}^{(1)}(0)\right\rangle d t\right\}_{\mathrm{sp} \mathrm{av}}
\end{aligned}
$$

The square brackets denote a trace over the electron-spin operators, and the curly brackets indicate a spatial average over the variables $(\vartheta, \varphi)$.

Choice of coordinate systems. The definition of the spherical tensor components $F_{q}^{(1)}$ as given by Eq. [9] is valid for any coordinate system, as long as the components of $\mathbf{S}^{(1)}$ and $\mathbf{C}^{(1)}$ are expressed in that same system. I will use the convention here that tensors and their components that are written without a superscripting caret (e.g., $\left.\mathbf{F}^{(1)}, \mathbf{I}^{(1)}, \mathbf{S}^{(1)}, \mathbf{C}^{(1)}\right)$ are defined in the laboratory frame, while those with a caret (e.g., $\hat{\boldsymbol{F}}^{(1)}$ ) are defined in the molecular coordinate system which diagonalizes the $\mathrm{zfs}$ tensor. The components of a tensor that are defined in one coordinate system can be expressed in any other frame by a transformation involving the Wigner rotation-matrix elements, $\mathcal{D}_{q}^{(1), q}(\alpha, \beta, \gamma)$, where $\alpha, \beta, \gamma$ are the Euler angles that rotate the original frame to the final frame; for example,

$$
F_{q}^{(1)}=\sum_{q^{\prime}} \hat{F}_{q^{\prime}}^{(1)} \mathcal{D}_{q^{\prime}, q}^{(1)}(\alpha, \beta, \gamma)
$$

[The standard texts on spherical tensor methods unfortunately differ to some degree in symbolism, definitions, and phase conventions. I have followed the treatment of Silver (23) throughout.]

The tensor operators in Eqs. [2], [5], [6], [9], [11], and [12] are expressed entirely in the laboratory coordinate frame. This is always the most convenient choice for the nuclear-spin variables, $I_{q}^{(1)}$, since the motion of the spin vector $I$ is quantized by the laboratory magnetic field, $\mathbf{B}_{0}$. When the motion of the electron-spin vector $\mathbf{S}$ is in the Zeeman limit, this frame is also the most convenient choice for the components of S. The choice of coordinate system for the components of $\mathbf{C}^{(2)}$ is irrelevant in the 
Zeeman limit, since nuclear-spin relaxation and $\mathscr{H}_{\mathrm{S}}$ are in this situation independent of the orientation of the I-S vector in the molecular system. The spatial variables $(\vartheta$, $\phi$ ) occur only in the time correlation function $\mathcal{C}(t) \equiv\left\{C_{q}^{(2)}(t) C_{q}^{(2) *}(0)\right\}_{\text {sp av }}$, not in the spin Hamiltonian. In an isotropic medium, $\mathcal{C}(t)$ is independent of the coordinate system:

$$
\mathcal{C}(t)=\left\{\left|C_{q}^{(2)}(\vartheta, \phi)\right|^{2}\right\}_{\mathrm{sp} \mathrm{av}} \exp \left(-t / \tau_{\mathrm{R}}^{(2)}\right)=5{ }^{1} \exp \left(-t / \tau_{\mathrm{R}}^{(2)}\right) .
$$

In the Zeeman limit, the laboratory frame is the most convenient choice for evaluating Eqs. [11] and [12].

In the zfs limit, the electron-spin angular momentum is quantized along molecular axes, and the tensor operators $S^{(j)}$ (particularly the time dependence of these operators) can be expressed most simply in the molecular frame. In this situation, it is also convenient to express the tensor functions $C_{q}^{(2)}(\vartheta, \phi)$ in the molecular coordinate frame,

$$
C_{q}^{(2)}(\vartheta, \phi)=\sum_{q^{\prime}} \hat{C}_{q^{\prime}}^{(2)}(\theta, \vartheta) \mathcal{D}_{q^{\prime}, q}^{(2)}(\alpha, \beta, \gamma)
$$

since $\left(T_{1 p}\right)_{\text {dip }}^{-1}$ and $\left(T_{2 p}\right)_{\text {dip }}^{-1}$ depend on the orientation of the I-S vector with respect to the principal axes of the zfs tensor. The functions $\hat{C}_{q}^{(2)}(\theta, \varphi)$ specify the orientation of the I-S vector in the molecular frame $[(\theta, \varphi)$ are polar angles of the I-S vector]. For rigid molecules these functions are independent of molecular orientation. When both $S^{(j)}$ and $C^{(2)}$ are expressed in the molecular frame, an equivalent and computationally simpler procedure is to express the compound tensor $\hat{\boldsymbol{F}}^{(1)}$ in the molecular frame using Eq. [13], where

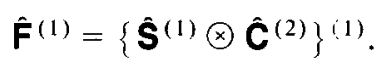

This latter procedure was used previously to derive $\mathrm{zfs}$-limit expressions for $\left(T_{1 p}\right)_{\mathrm{dip}}^{-1}$ and $\left(T_{2 p}\right)_{\text {dip }}^{-1}(5,6)$.

The present objective is to cast Eqs. [11] and [12] in a form that is suitable for numerical evaluation in the intermediate regime, where both $\mathscr{H}_{\mathrm{Z}}$ and $\mathscr{H}_{\text {zfs }}$ are appreciable. For this purpose, the components of $\mathrm{I}^{(1)}$ are best expressed in the laboratory frame and those of $\mathbf{C}^{(2)}$ in the molecular frame. The electron-spin operators occur in Eqs. [11] and [12] in the form of a time correlation function, $\operatorname{Tr}\left\{S_{p}^{(1)}(t) S_{p^{\prime}}^{(1)}(0)\right\}$. This trace can be evaluated numerically in either the Zeeman or zfs basis. In the following calculations, the Zeeman basis will be used since it is generally more familiar and since the resulting expressionsfor $T_{1 p}^{-1}$ then extrapolate very simply to the SBM equations.

Summarizing, the tensor operators $I_{q}^{(1)}, F_{q}^{(1)}$, and $S_{q}^{(1)}$ will be expressed in the laboratory coordinate frame and the functions $\hat{C}_{q}^{(\%)}(\theta, \varphi)$ in the molecular frame. This gives, for the terms of the integrand of Eq. [11],

$$
\begin{aligned}
& \left\langle F_{ \pm 1}^{(1)}(t) F_{+1}^{(1)}(0)\right\rangle e^{\mp i \omega_{1} t}=\sum_{p, p^{\prime}} c_{ \pm 1, p} c_{\mp 1, p^{\prime}} \sum_{q, q^{\prime}} \hat{C}_{q}^{(2)}(\theta, \varphi) \hat{C}_{q^{\prime}}^{(2)}(\theta, \varphi) \\
& \quad \times \mathcal{D}_{q, \pm 1-p}^{(2)}\left(\alpha_{1}, \beta_{1}, \gamma_{1} ; t\right) \mathcal{D}_{q^{\prime}, \mp 1-p^{\prime}}^{(2)}(\alpha, \beta, \gamma ; 0) \operatorname{Tr}\left\{S_{p}^{(1)}(t) S_{p^{\prime}}^{(1,)}(0)\right\} e^{\mp i \omega_{1} I^{\prime}},
\end{aligned}
$$

where $(\alpha, \beta, \gamma)$ and $\left(\alpha_{1}, \beta_{1}, \gamma_{1}\right)$ are the Euler angles which take the laboratory frame into the molecular frame at time zero and $t$, respectively.

In accord with the assumption that molecular reorientation is slow relative to electron-spin relaxation, we can write 


$$
\mathcal{D}_{l, l^{\prime}}^{(2)}\left(\alpha_{1}, \beta_{1}, \gamma_{1} ; t\right) \cong \mathcal{D}_{l, l^{\prime}}^{(2)}(\alpha, \beta, \gamma ; 0)
$$

Thus, the entire time dependence of Eq. [17] occurs in the spin variables, in the form of time correlation functions

$$
\begin{aligned}
& M_{p, p^{\prime}}^{( \pm)}\left(\alpha, \beta, \gamma ; \alpha_{1}, \beta_{1}, \gamma_{1} ; t\right) \equiv e^{ \pm i \omega_{\mathrm{I}} t} \operatorname{Tr}\left\{S_{p}^{(1)}(t) S_{p^{\prime}}^{(1)}(0)\right\}
\end{aligned}
$$

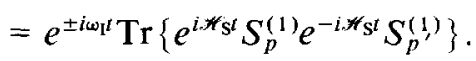

The trace over the electron-spin variables is in general a function of the Euler angles relating the molecular and laboratory frames because of the spatial dependence of $\mathscr{H}_{\mathrm{S}}$. In fact the trace depends on two sets of Euler angles, those at time zero $(\alpha, \beta, \gamma)$ and those at time $t\left(\alpha_{1}, \beta_{1}, \gamma_{1}\right)$. From our assumption of slow motion $\left(\tau_{\mathbf{R}} \gg \tau_{\mathrm{S}}\right)$, however, $M_{p, p}^{( \pm)}$can be considered a function only of a single set of Euler angles and written $M_{p, p}^{( \pm)},(\alpha, \beta, \gamma ; t)$. In the following discussion, the Euler angles and the time variable are generally omitted for brevity, with the functions $\mathcal{D}_{l, l^{\prime}}^{(2)}$ and $M_{p, p}^{( \pm)}$, used as shorthand abbreviations for those in Eqs. [18] and [19].

This gives the following expression for $\left(T_{1 p}\right)_{\mathrm{dip}}^{-1}$ :

$$
\left(T_{1 p}\right)_{\mathrm{dip}}^{-1}=(-1)\left(\frac{3^{-1 / 2} \kappa}{r^{3}}\right)^{2}\left\{\int_{0}^{\infty} \Theta_{1}(\alpha, \beta, \gamma ; t) d t\right\}_{\mathrm{sp} \mathrm{av}}
$$

with

$$
\begin{aligned}
\Theta_{1}(\alpha, \beta, \gamma ; t) & \equiv\left\langle F_{+1}^{(1)}(t) F_{-1}^{(1)}(0)\right\rangle e^{-i \omega_{\mathrm{I}} t}+\left\langle F_{-1}^{(1)}(t) F_{+1}^{(1)}(\theta)\right\rangle e^{i \omega_{1} t} \\
& =\sum_{p, p^{\prime}} c_{+1, p} c_{-1, p^{\prime}} \sum_{q, q^{\prime}} \hat{C}_{q}^{(2)} \hat{C}_{q^{\prime}}^{(2)} D_{q,-1-p}^{(2)} D_{q^{\prime},+1-p^{\prime}}^{(2)}\left[M_{p, p^{\prime}}^{(-)}+M_{p^{\prime}, p}^{(+)}\right] .
\end{aligned}
$$

The quadruple summations in Eq. [21] comprise a rather formidable expression containing 450 terms (since $p, p^{\prime}=-1,0,+1$ and $q, q^{\prime}=-2,-1,0,+1,+2$ ). However it can be shown from a detailed consideration of the spatial dependence of the time correlation functions $M_{p, p^{\prime}}^{(+)}$and $M_{p, p^{\prime}}^{(-)}$that when the zfs tensor possesses uniaxial symmetry, all terms of Eq. [21] vanish except those for which $q^{\prime}=-q$ (see Appendix A). There is no similar restriction on the indices $p, p^{\prime}$.

Using the condition $q=-q^{\prime}$, expanding the summation over $p, p^{\prime}$, and inserting numerical values for the coefficients $c_{+1, p} c_{-1, p^{\prime}}$ give

$$
\begin{aligned}
& \Theta_{1}(\alpha, \beta, \gamma ; t)=10^{-1} \sum_{q} \hat{C}_{q}^{(2)} \hat{C}_{-q}^{(2)} \\
& \times\left\{6\left(D_{q,+2}^{(2)} D_{-q,-2}^{(2)} M_{-1,+1}^{(+)}+D_{q,-2}^{(2)} D_{q,+2}^{(2)} M_{+1,-1}^{(-)}\right)\right. \\
& +3\left(\mathcal{D}_{q,+1}^{(2)} \mathcal{D}_{-q,-1}^{(2)} M_{0,0}^{(-)}+\mathscr{D}_{q,-1}^{(2)} \mathcal{D}_{q,+1}^{(2)} M_{0,0}^{(+)}\right)+\mathscr{D}_{q, 0}^{(2)} \mathcal{D}_{-q, 0}^{(2)}\left(M_{-1,+1}^{(+)}+M_{+1,-1}^{(-)}\right) \\
& +6^{1 / 2}\left(D_{q, 0}^{(2)} D_{-q,-2}^{(2)} M_{+1,+1}^{(-)}+D_{q,-2}^{(2)} D_{-q, 0}^{(2)} M_{+1,+1}^{(+)}\right. \\
& \left.\times \mathscr{D}_{q, 0}^{(2)} \mathscr{D}_{-q,+2}^{(2)} M_{-1,-1}^{(-)}+\mathcal{D}_{q,+2}^{(2)} \mathcal{D}_{-q, 0}^{(2)} M_{-1,-1}^{(+)}\right) \\
& -3^{1 / 2}\left(D_{q, 0}^{(2)} D_{-q,-1}^{(2)} M_{+1,0}^{(-)}+D_{q,-1}^{(2)} D_{-q, 0}^{(2)} M_{0,+1}^{(+)}\right. \\
& \times \mathcal{D}_{q,+1}^{(2)} \mathcal{D}_{-q, 0}^{(2)} M_{0,-1}^{(-)}+\mathcal{D}_{q, 0}^{(2)} \mathcal{D}_{-q,+1}^{(2)} M_{-1,0}^{(+)} \\
& -18^{1 / 2}\left(\mathcal{D}_{q,-2}^{(2)} \mathcal{D}_{-q,+1}^{(2)} M_{+1,0}^{(+)}+\mathcal{D}_{q,+1}^{(2)} \mathcal{D}_{-q,-2}^{(2)} M_{0,+1}^{(-)}\right. \\
& \left.\left.+\mathcal{D}_{q,-1}^{(2)} \mathcal{D}_{-q,+2}^{(2)} M_{0,-1}^{(+)}+\mathcal{D}_{q,+2}^{(2)} \mathcal{D}_{-q,-1}^{(2)} M_{-1,0}^{(-)}\right)\right\} .
\end{aligned}
$$


This expression can be further simplified by contracting the pairwise products of rotation-matrix elements, $\mathscr{D}_{q, l}^{(2)} \mathscr{D}_{-q, l^{\prime}}^{(2)}$, to a sum of terms, each containing a single rotationmatrix element, by using the Clebsch-Gordan formula

$$
\begin{aligned}
& \mathcal{D}_{m_{1}^{\prime}, m_{1}}^{\left(j_{1}\right)}(\alpha \beta \gamma) \mathcal{D}_{m_{2}, m_{2}}^{\left(j_{2}\right)}(\alpha \beta \gamma) \\
& \quad=\sum_{j}(2 j+1)(-1)^{m^{\prime}+m}\left[\begin{array}{ccc}
j_{1} & j_{2} & j \\
m_{1}^{\prime} & m_{2}^{\prime} & -m^{\prime}
\end{array}\right]\left[\begin{array}{ccc}
j_{1} & j_{2} & j \\
m_{1} & m_{2} & -m
\end{array}\right] \mathcal{D}_{m^{\prime}, m}^{(j)}(\alpha \beta \gamma),
\end{aligned}
$$

where $m=m_{1}+m_{2}$ and $m^{\prime}=m_{1}^{\prime}+m_{2}^{\prime}$. The products $\hat{C}_{q}^{(2)} \hat{C}_{-q}^{(2)}$ can be contracted in an identical manner, using Eq. [7]. After laborious calculations, the results of which are summarized in Appendix $\mathrm{B}, \Theta_{1}$ finally reduces to a sum of terms, each of which is bilinear in one spherical tensor function of the (fixed) polar variables $(\theta, \varphi)$ that specify the orientation of the I-S vector in the molecular frame and one rotationmatrix element in the Euler angles $(\alpha, \beta, \gamma)$ which specify the orientation of the molecular coordinate frame in the laboratory system:

$$
\begin{aligned}
& \Theta_{1}(\alpha, \beta, \gamma ; t)=10^{-1}\left\{2 M_{-1,+1}^{( \pm)}\left[5^{-1}+\frac{2}{7} \hat{P}_{2}(\theta) P_{2}(\beta)+\frac{18}{35} \hat{P}_{4}(\theta) P_{4}(\beta)\right]\right. \\
& +3{ }^{1}{ }^{1} M_{0,0}^{( \pm)}\left[-5^{-1}-7^{-1} \hat{P}_{2}(\theta) P_{2}(\beta)+\frac{12}{35} \hat{P}_{4}(\theta) P_{4}(\beta)\right] \\
& +2{ }^{1} 6^{1} 2^{1} M_{-1,+1}^{( \pm)}\left[5^{-1}-\frac{2}{7} \hat{P}_{2}(\theta) P_{2}(\beta)+\frac{3}{35} \hat{P}_{4}(\theta) P_{4}(\beta)\right] \\
& -3^{1 / 2} 2^{1} M_{+1,0}^{( \pm)}\left[7^{-1} \hat{P}_{2}(\theta) D_{0,-1}^{(2)}(\alpha, \beta, \gamma)+\left(\frac{6}{5}\right)^{1 / 2} \frac{3}{7} \hat{P}_{4}(\theta) D_{0,-1}^{(4)}(\alpha, \beta, \gamma)\right] \\
& -2{ }^{1} 3^{1 / 2} M_{-1,0}^{( \pm)}\left[-7^{-1} \hat{P}_{2}(\theta) D_{++1,0}^{(2)}(\alpha, \beta, \gamma)-\left(\frac{6}{5}\right)^{1 / 2} \frac{3}{7} \hat{P}_{4}(\theta) D_{+1,0}^{(4)}(\alpha, \beta, \gamma)\right] \\
& +6{ }^{1 / 2} 2^{1} M_{+1,+1}^{( \pm)}\left[-\frac{2}{7} \hat{P}_{2}(\theta) D_{0,-2}^{(2)}(\alpha, \beta, \gamma)+\left(\frac{3}{5}\right)^{1 / 2} \frac{3}{7} \hat{P}_{4}(\theta) D_{0,-2}^{(4)}(\alpha, \beta, \gamma)\right] \\
& +2{ }^{1} 6^{1 / 2} M_{-1,-1}^{( \pm)}\left[-\frac{2}{7} \hat{P}_{2}(\theta) D_{0,+2}^{(2)}(\alpha, \beta, \gamma)+\left(\frac{3}{5}\right)^{1 / 2} \frac{3}{7} \hat{P}_{4}(\theta) D_{0,+2}^{(4)}(\alpha, \beta, \gamma)\right] \\
& -18^{1 / 2} 2^{1} M_{+1,0}^{( \pm)}\left[-6{ }^{1 / 2} 7^{-1} \hat{P}_{2}(\theta) D_{0,-1}^{(2)}(\alpha, \beta, \gamma)+5^{-1 / 2} \frac{3}{7} \hat{P}_{4}(\theta) D_{0,-1}^{(4)}(\alpha, \beta, \gamma)\right] \\
& -18^{1 / 2} 2^{1} M_{-1,0}^{( \pm)}\left[-6^{1 / 2} 7^{-1} \hat{P}_{2}(\theta) D_{0,+1}^{(2)}(\alpha, \beta, \gamma)+5^{-1 / 2} \frac{3}{7} \hat{P}_{4}(\theta) D_{0,+1}^{(4)}(\alpha, \beta, \gamma)\right]
\end{aligned}
$$

The functions $P_{n}(\theta)$ and $P_{n}(\beta)$ are Legendre polynomials of order $n$ in $\cos \theta$, where $\theta$ is (the polar angle of the I-S vector in the molecular frame) and $\cos \beta$, where $\beta$ is an Euler angle. Legendre polynomials appear in Eq. [24] due to the fact that they are a special form of the Wigner rotation-matrix elements, $D_{0,0}^{(n)}(\alpha, \beta, \gamma)=P_{n}(\beta)$ and result from applications of the Clebsch-Gordan formula (Eq. [23]) to rotation-matrix products of the form $\mathcal{D}_{m, m}^{(n)}(\alpha, \beta, \gamma) \mathcal{D}_{-m,-m^{\prime}}^{(n)}(\alpha, \beta, \gamma)$.

To evaluate $\left(T_{1 p}\right)_{\text {dip }}^{-1}$ by Eq. [20], $\Theta_{1}(\alpha, \beta, \gamma ; t)$ must first be integrated over time and then averaged over molecular orientations, i.e., over the space of the Euler angles $(\alpha, \beta, \gamma) . \Theta_{1}(\alpha, \beta, \gamma ; t)$ depends on molecular orientation explicitly through the rotation-matrix elements and implicitly through the time correlation functions, $M_{p, p}^{(\perp)}(t)$. Prior to calculating the spatial average, it will be convenient to simplify $\Theta_{1}(\alpha, \beta, \gamma ; t)$ still further using a number of symmetric relationships which can be shown to exist among the $M_{p, p}^{( \pm)},(t)$ and their Fourier transforms. The evaluation of 
these functions and the relations among them are described in the following two sections.

Evaluation of the time correlation functions, $M_{p, p^{\prime}}^{( \pm)}(t)$. The time dependence of $\Theta_{1}(\alpha$, $\beta, \gamma ; t)$ resides entirely in the time correlation functions, $M_{p, p^{\prime}}^{( \pm)}(t)$, defincd in Eq. [19]. The evaluation of these functions is greatly simplified when molecular reorientation is slow on the time scale of electron-spin relaxation. In this situation, $\mathscr{H}_{\mathrm{S}}$ can be written as a sum of two terms:

$$
\mathscr{H}_{\mathrm{S}}=\mathscr{H}_{\mathrm{S}}^{(0)}(\alpha, \beta, \gamma)+\mathscr{H}_{\mathrm{S}}^{(1)}(t) .
$$

$\mathscr{H}_{\mathrm{S}}^{(0)}$ describes the precessional motion of the spin due to the Zeeman and zfs interactions:

$$
\mathscr{H}_{\mathrm{S}}^{(0)}=g \beta_{0} B_{0} S_{0}^{(1)}+\left(\frac{2}{3}\right)^{1 / 2} D \hat{S}_{0}^{(2)}+E\left(\hat{S}_{+2}^{(2)}+\hat{S}_{-2}^{(2)}\right) .
$$

$\mathscr{H}_{\mathrm{S}}^{(1)}(t)$ represents the time-dependent couplings of the electron spin with the thermal lattice that give rise to electron-spin relaxation. An assumption of uniaxial symmetry in the zfs tensor $(E=0)$ was adopted implicitly above when the restriction $q=-q^{\prime}$ was applied to Eq. [22] (see Appendix A). Relaxation of this assumption leads to a considerable increase in complexity in the final expressions and will not be considered here. The effects of $\mathscr{H}_{\mathrm{S}}^{(1)}(t)$ will be described phenomenologically through the use of an exponential term $\exp \left(-t / \tau_{\mathrm{S}}\right)$ containing as a parameter a single relaxation time $\tau_{\mathrm{S}}$ in the time correlation functions $M_{p, p}^{( \pm)},(t)$.

It should be noted that the spin operators in Eq. [26] are written in different coordinate frames, $S_{0}^{(1)}$ in the laboratory frame and $\hat{S}_{0}^{(2)}$ in the molecular frame. $\mathscr{H}_{\mathrm{S}}^{(0)}$ can be written in a single coordinate frame by expanding $\hat{S}_{0}^{(2)}$ in the laboratory components $S_{q}^{(2)}$. This transformation is the inverse of that used above for the functions $C_{q}^{(2)}$ (Eq. [15]); i.e., the components in the molecular frame are being expressed in the laboratory system rather than vice versa. Written in the laboratory system, $\mathscr{H}_{\mathrm{S}}^{(0)}$ has the form

$$
\mathscr{H}_{\mathrm{S}}^{(0)}=g \beta_{0} B_{0} S_{0}^{(1)}+\left(\frac{2}{3}\right)^{1 / 2} D \sum_{q=-2}^{2} S_{q}^{(2)}\left[D_{q, 0}^{(2)}(\alpha, \beta, \gamma)\right]^{-1},
$$

where the functions $\left[\mathscr{D}_{q, 0}^{(j)}(\alpha, \beta, \gamma)\right]^{-1}$ are matrix elements of the inverse transformation. They can be written in terms of the functions $D_{q, q^{\prime}}^{(2)}(\alpha, \beta, \gamma)$ through the identities $(23,24)$

$$
\begin{aligned}
{\left[\mathscr{D}_{q, q^{\prime}}^{(j)}(\alpha, \beta, \gamma)\right]^{-1} } & =\mathscr{D}_{q, q^{\prime}}^{(j)}(-\gamma,-\beta,-\alpha) \\
& =\left[\mathcal{D}_{q^{\prime}, q}^{(j)}(\alpha, \beta, \gamma)\right]^{*}=(-1)^{q-q^{\prime}} \mathcal{D}_{-q^{\prime},-q}^{(j)}(\alpha, \beta, \gamma)
\end{aligned}
$$

This gives

$$
\mathscr{H}_{\mathrm{S}}^{(0)}=g \beta_{0} B_{0} S_{0}^{(1)}+\left(\frac{2}{3}\right)^{1 / 2} D \sum_{q=-2}^{2}(-1)^{q} S_{q}^{(2)} \mathscr{D}_{0,-q}^{(2)}(\alpha, \beta, \gamma)
$$

In keeping with the assumption that $\tau_{\mathrm{R}} \gg \tau_{\mathrm{S}}, \mathscr{H}_{\mathrm{S}}^{(0)}$ can be treated as static over the time interval during which $M_{p, p^{\prime}}^{( \pm)}(t)$ is appreciable. The trace over the operators of $\mathbf{S}$ (Eq. [19]) can be evaluated in the Heisenberg representation by setting 


$$
\hat{S}_{p}^{(1)}(t) \hat{S}_{p^{\prime}}^{(1)}(0)=\exp \left(i \mathscr{H}_{\mathrm{S}}^{(0)} t\right) \hat{S}_{p}^{(1)} \exp \left(-i \mathscr{H}_{\mathrm{S}}^{(0)} t\right) \hat{S}_{p^{\prime}}^{(1)} \exp \left(-t / \tau_{\mathrm{S}}\right)
$$

and evaluating the trace in the basis set comprised of the eigenvectors of $\mathscr{H}_{\mathrm{S}}^{(0)}$. In this basis, the matrix elements of $\hat{S}_{p}^{(1)}(t)$ can be written $\left\langle\mu\left|\hat{S}_{p}^{(1)}(t)\right| \nu\right\rangle=e^{i\left(\omega_{\mu}-\omega_{\nu}\right) t}$ $\left\langle\mu\left|\hat{S}_{p}^{(1)}\right| \nu\right\rangle$, where $\omega_{\mu}, \omega_{\nu}$ are eigenvalues associated with the eigenvectors $|\mu\rangle,|\nu\rangle$. In the high-temperaiure limit for $\mathbf{S}$, the trace becomes

$$
\operatorname{Tr}\left\{\hat{S}_{p}^{(1)}(t) \hat{S}_{p^{\prime}}^{(1)}(0)\right\}=(2 S+1)^{-1} \sum_{\mu, \nu} e^{i\left(\omega_{\mu^{\prime}}-\omega_{\nu}\right) t} e^{-t / \tau \mathrm{s}}\left\langle\mu\left|\hat{S}_{p}^{(1)}\right| \nu\right\rangle\left\langle\nu\left|\hat{S}_{p^{\prime}}^{(1)}\right| \mu\right\rangle,
$$

giving

$$
\begin{aligned}
M_{p, p^{\prime}}^{( \pm)}(t) & =e^{ \pm i \omega_{l} t} \sum_{\mu, \nu}\left\langle\mu\left|e^{i: *_{\mathrm{S}}^{(0)} t}\right| \mu\right\rangle\left\langle\mu\left|S_{p}^{(1)}\right| \nu\right\rangle\left\langle\nu\left|e^{-i \nVdash \varkappa_{\mathrm{s}}^{(0)}}\right| \nu\right\rangle\left\langle\nu\left|S_{p^{\prime}}^{(1)}\right| \mu\right\rangle \\
& =\sum_{\mu, \nu}\left\langle\mu\left|S_{p}^{(1)}\right| \nu\right\rangle\left\langle\nu\left|S_{p}^{(1)}\right| \mu\right\rangle e^{i\left(\omega_{\mu}-\omega_{\nu} \pm \omega_{1}\right) t} e^{-t / \tau_{\mathrm{s}}} .
\end{aligned}
$$

Equation [33] exhibits the time dependence of $M_{p, p^{\prime}}^{( \pm)},(t)$ in explicit form. The integration with respect to time in Eq. [20] can now be carried out analytically, giving

$$
\begin{aligned}
m_{p, p^{\prime}}^{( \pm)} & \equiv \int_{0}^{\infty} M_{p, p^{( \pm)}}^{( \pm)}(t) d t \\
& =\sum_{\mu, \nu}\left\langle\mu\left|S_{p}^{(1)}\right| \nu\right\rangle\left\langle\nu\left|S_{p^{\prime}}^{(1)}\right| \mu\right\rangle j\left(\omega_{\mu}-\omega_{\nu} \pm \omega_{\mathrm{I}}\right)
\end{aligned}
$$

with $j(\omega)=\tau_{\mathrm{S}}\left(1+\omega^{2} \tau_{\mathrm{S}}^{2}\right)^{-1}$. Because of their dependence on $\mathscr{H}_{\mathrm{S}}^{(0)}$, the $m_{p, p^{\prime}}^{( \pm)}$, depend implicitly on the Euler angles $(\alpha, \beta, \gamma)$.

Symmetric relationships among the $m_{p, p}^{( \pm)}$. A number of symmetries exist among the factors $m_{p, p}^{( \pm)}$, in the approximation (which is always very good) that $\omega_{\mathrm{I}}$ is small compared to the transition frequencies of the electron-spin system. These symmetries provide important simplifications of the final results and are summarized here.

The functions $j_{p, p^{\prime}}\left(\omega_{\mu}-\omega_{\nu} \pm \omega_{\mathrm{I}}\right)$ can, to a high degree of accuracy, be considered symmetric in $\pm \omega_{1}$. When $\mu \neq \nu$,

$$
j_{p, p^{\prime}}\left(\omega_{\mu}-\omega_{\nu} \pm \omega_{1}\right) \cong j_{p, p^{\prime}}\left(\omega_{\mu}-\omega_{\nu}\right),
$$

and when $\mu=\nu$,

$$
j_{p, p^{\prime}}\left(\omega_{\mu}-\omega_{\nu} \pm \omega_{\mathrm{I}}\right)=j_{p, p^{\prime}}\left( \pm \omega_{\mathrm{I}}\right)=j_{p, p^{\prime}}\left(\omega_{\mathrm{I}}\right) .
$$

Thus the superscripts on $m_{p, p^{\prime}}^{(+)}$and $m_{p, p^{\prime}}^{(-)}$will be denoted simply $( \pm)$. To the same (high) degree of approximation, Eq. [34b] is symmetric in $p$ and $p^{\prime}$; i.e., $m_{p, p}^{( \pm)}$, $=m_{p}^{\left(\frac{ \pm}{\prime}\right)}$.

Additional simplifying relationships result from symmetries among the matrix elements $\left\langle\mu\left|S_{p}^{(1)}\right| \nu\right\rangle$. From the properties of the spherical tensor components, $S_{0}^{(1)}$ is self-adjoint, while $-\left(S_{+1}^{(1)}\right)^{\dagger}=S_{-1}^{(1)}$. Thus

$$
\left\langle\mu\left|S_{0}^{(1)}\right| \nu\right\rangle=\left(\left\langle\nu\left|S_{0}^{(1)}\right| \mu\right\rangle\right)^{*}
$$

and

$$
-\left\langle\mu\left|S_{+1}^{(1)}\right| \nu\right\rangle=\left(\left\langle\nu\left|S_{-1}^{(1)}\right| \mu\right\rangle\right)^{*} .
$$


Using these relations, along with the fact that $j\left(\omega_{\mu}-\omega_{v}\right)=j\left(\omega_{v}-\omega_{\mu}\right)$, it is readily shown that

$$
\begin{aligned}
& m_{+1,-1}^{( \pm)}=\sum_{\mu, \nu}\left\langle\mu\left|S_{+1}^{(1)}\right| \nu\right\rangle\left\langle\nu\left|S_{-1}^{(1)}\right| \mu\right\rangle j\left(\omega_{\mu}-\omega_{\nu}\right)=m_{-1,11}^{( \pm)} \\
& m_{+1,0}^{( \pm)}=\sum_{\mu, \nu}\left\langle\mu\left|S_{+1}^{(1)}\right| \nu\right\rangle\left\langle\nu\left|S_{0}^{(1)}\right| \mu\right\rangle j\left(\omega_{\mu}-\omega_{\nu}\right)=\left(m_{-1,0}^{( \pm)}\right)^{*}
\end{aligned}
$$

and

$$
m_{+1,+1}^{(+)}=\sum_{\mu, \nu}\left\langle\mu\left|S_{+1}^{(1)}\right| \nu\right\rangle\left\langle\nu\left|S_{+1}^{(1)}\right| \mu\right\rangle j\left(\omega_{\mu}-\omega_{\nu}\right)=\left(m_{-1,-1}^{( \pm)}\right)^{*}
$$

Simplification of the algebraic form of $\Theta_{1}(\alpha, \beta, \gamma)$. These relations can be used to simplify, very substantially, the final expressions for $\left(T_{1 p}\right)^{-1}$, which reduce to

$$
\begin{aligned}
& \tilde{\Theta}_{1}(\alpha, \beta, \gamma) \equiv \int_{0}^{\infty} \Theta_{1}(\alpha, \beta, \gamma ; t) d t=10^{-1}\left\{6 m _ { 0 , 0 } ^ { ( \pm ) } ( \beta ) \left[-5^{-1}-7^{-1} \hat{P}_{2}(\theta) P_{2}(\beta)\right.\right. \\
& \left.+\frac{12}{35} \hat{P}_{4}(\theta) P_{4}(\beta)\right]+2 m_{+1,-1}^{( \pm)}(\beta)\left[+\frac{7}{5}-\frac{10}{7} \hat{P}_{2}(\theta) P_{2}(\beta)+\frac{36}{35} \hat{P}_{4}(\theta) P_{4}(\beta)\right] \\
& +2 \times 6^{1 / 2}\left[-\frac{2}{7} \hat{P}_{2}(\theta)\left(\mathcal{D}_{0,2}^{(2)}(\alpha, \beta, \gamma) m_{11,11}^{( \pm)}(\alpha, \beta, \gamma)+\text { c.c. }\right)\right. \\
& \left.+\left(\frac{3}{5}\right)^{1 / 2} \frac{12}{35} \hat{P}_{4}(\theta)\left(\mathcal{D}_{0,-2}^{(4)}(\alpha, \beta, \gamma) m_{+1,+1}^{( \pm)}(\alpha, \beta, \gamma)+\text { c.c. }\right)\right] \\
& -2\left[-3^{1 / 2} \frac{5}{7} \hat{P}_{2}(\theta)\left(D_{0,-1}^{(2)}(\alpha, \beta, \gamma) m_{+1,0}^{( \pm)}(\alpha, \beta, \gamma)+\text { c.c. }\right)\right. \\
& \left.\left.+\left(\frac{2}{5}\right)^{1 / 2} \frac{18}{7} \hat{P}_{4}(\theta)\left(D_{0,-1}^{(4)}(\alpha, \beta, \gamma) m_{++1,0}^{( \pm)}(\alpha, \beta, \gamma)+\text { c.c. }\right)\right]\right\} .
\end{aligned}
$$

The final four terms in this expression have been simplified using Eqs. [38], [39], and [21]. Algebraic forms of the rotation-matrix elements are tabulated in Appendix $D$.

Equation [40] is suitable for numerical evaluation of $\tilde{\Theta}_{1}(\alpha, \beta, \gamma)$. This involves diagonalization of $\mathscr{H}_{\mathrm{s}}^{(0)}$ in the Zeeman basis as a function of molecular orientation (i.e., of the Euler angles) and then, using the eigensystem of the electron spin, calculation of the $m_{p, p}^{( \pm)},(\alpha, \beta, \gamma)$.

Calculation of the spatial average of $\tilde{\theta}_{1}(\alpha, \beta, \gamma)$. In the derivation of Eq. [40] an assumption of uniaxial symmetry in the zfs tensor was employed. This assumption greatly simplifies not only the multiple summations of Eq. [21], but also the calculation of the spatial average of Eq. [20], since in this situation $\tilde{\Theta}_{1}$ is a function only of the polar angle $\beta$ between the $z$ and $\hat{z}$ axes ( see Appendix $C$ ). Thus the spatial average of $\tilde{\Theta}_{1}(\beta)$ can be calculated simply from integration over the space of the polar angle $\beta$ :

$$
\left(T_{1 p}\right)_{\mathrm{dip}}^{-1}=(-1)\left(\frac{3^{-1 / 2} \kappa}{r^{3}}\right)^{2} 2^{-1} \int_{0}^{\pi} \tilde{\Theta}_{1}(\beta) \sin (\beta) d \beta .
$$




\section{$T_{2}$ RELAXATION}

According to Eq. [11], the calculation of the dipole portion of $\left(T_{1 p}\right)_{\text {dip }}^{-1}$ due to coupling to a paramagnetic spin depends on the transverse components of the dipolar interaction, through the sum of terms $\left[I_{+1}^{(1)}(t) F_{-1}^{(1)}(t)+I_{-1}^{(1)}(t) F_{+1}^{(1)}(t)\right]$. The calculation of $\left(T_{2 p}\right)_{\text {dip }}^{-1}$ (Eq. [12]) depends on these terms and, additionally, on the coupling of the longitudinal components $I_{0}^{(1)} F_{0}^{(1)}(t)$. In the Zeeman limit, the $z$ components of $\mathbf{I}$ and $\mathbf{S}$ do not undergo time-dependent precessional motion; rather their time dependence is entirely stochastic, due to electron-spin relaxation and molecular reorientation. It is the longitudinal $I_{0}^{(1)} F_{0}^{(1)}(t)$ coupling that gives rise to "zero-frequency" terms proportional to $j(0)$ in SBM theory.

This physical picture changes outside the Zeeman limit, where the precessional motion of $\mathbf{S}$ is no longer quantized along the external magnetic field, but rather along quantization axes which depend both on the relative magnitudes of the zfs and Zeeman interactions and on the relative orientations of the molecular and laboratory axes. For this reason, the $I_{0}^{(1)} F_{0}^{(1)}(t)$ coupling gives rise to terms proportional to $j\left(\omega_{\mu}-\omega_{\nu}\right)$ as well to $j(0)$. As above, it will be assumed that the molecular axes can be considered fixed over times the order of $\tau_{\mathrm{S}}$ so that the precessional frequencies of $\mathbf{S}$ are constant, although an explicit function of molecular orientation.

Using Eqs. [8]-[10], the longitudinal coupling term in Eq. [12] can be expanded in the form

$$
F_{0}^{(1)}(t) F_{0}^{(1)}(0)=\sum_{p, p^{\prime}} c_{0, p} c_{0, p^{\prime}} \sum_{q} \hat{C}_{q}^{(2)} \hat{C}_{-q}^{(2)} D_{q,-p}^{(2)} D_{-q,-p^{\prime}}^{(2)} M_{p, p^{\prime}}^{(0)}(t)
$$

where the choice of coordinate systems is the same as that used above for $\left(T_{1 p}\right)_{\text {dip. }}^{-1}$. Unlike the corresponding functions $M_{p, p^{\prime}}^{( \pm)}(t)$, the time correlation functions $M_{p, p}^{(0)},(t)$ are independent of the Larmor frequency of $\mathbf{I}$, a fact that is denoted by a superscript zero. Expanding the sum over the indices $p, p^{\prime}$ and inserting numerical values for the factors $c_{0, p}$ gives

$$
\begin{aligned}
& F_{0}^{(1)}(t) F_{0}^{(1)}(0)=10^{-1} \sum_{a} \hat{C}_{q}^{(2)} \hat{C}_{-q}^{(2)} \times\left\{+3\left[\mathcal{D}_{q,-1}^{(2)} D_{q,-1}^{(2)} M_{+1,+1}^{(0)}(t)\right.\right. \\
& \left.+\mathscr{D}_{q,-1}^{(2)} \mathcal{D}_{q,-1}^{(2)} M_{-1,-1}^{(0)}(t)\right]+4\left[\mathcal{D}_{q, 0}^{(2)} \mathcal{D}_{-q, 0}^{(2)} M_{0,0}^{(0)}(t)\right] \\
& +3\left[\mathcal{D}_{q,-1}^{(2)} \mathcal{D}_{-q,+1}^{(2)} M_{+1,-1}^{(0)}(t)+\mathcal{D}_{q,-1}^{(2)} \mathcal{D}_{-q,+1}^{(2)} M_{-1,+1}^{(0)}(t)\right] \\
& -12^{1 / 2}\left[\mathcal{D}_{q,-1}^{(2)} \mathcal{D}_{-q, 0}^{(2)} M_{+1,0}^{(0)}(t)+\mathcal{D}_{q, 0}^{(2)} \mathcal{D}_{-q,-1}^{(2)} M_{0,+1}^{(0)}(t)\right] \\
& \left.\quad-12^{1 / 2}\left[\mathcal{D}_{q, 0}^{(2)} \mathscr{D}_{-q,+1}^{(2)} M_{0,-1}^{(0)}(t)+\mathcal{D}_{q,+1}^{(2)} \mathcal{D}_{-q, 0}^{(2)} M_{-1,0}^{(0)}(t)\right]\right\} .
\end{aligned}
$$

This expression can be simplified greatly through the same procedures that were employed above in the calculation of $\left(T_{1 p}\right)_{\text {dip }}^{-1}$. First the pairwise products of tensor components can be contracted using the Clebsch-Gordan relation, Eq. [23]. The contracted forms are tabulated in Appendix B. Further simplification can be achieved using equivalence relations among the $M_{p, p}^{(0)}(t)$, i.e., Eqs. [37]-[39], along with Eq. [41]. After these simplifications, the final expression for $\left(T_{2 p}\right)_{\mathrm{dip}}^{-1}$ is

$$
\left(T_{2 p}\right)_{\mathrm{dip}}^{-1}=2^{-1}\left(T_{1 p}\right)_{\mathrm{dip}}^{-1}+3^{-1}\left(\frac{\kappa}{r^{3}}\right)^{2} 2^{-1} \int_{0}^{\pi} \tilde{\Theta}_{2}(\beta) \sin (\beta) d \beta
$$


with

$$
\begin{aligned}
& \tilde{\Theta}_{2}(\beta)=+4 m_{0,0}^{(0)}(\beta)\left[\left(\frac{1}{5}\right)+\left(\frac{2}{7}\right) \hat{P}_{2}(\theta) P_{2}(\beta)+\left(\frac{18}{35}\right) \hat{P}_{4}(\theta) P_{4}(\beta)\right] \\
& +6 m_{+1,-1}^{(0)}(\beta)\left[-\left(\frac{1}{5}\right)-\left(\frac{1}{7}\right) \hat{P}_{2}(\theta) P_{2}(\beta)+\left(\frac{12}{35}\right) \hat{P}_{4}(\theta) P_{4}(\beta)\right] \\
& +3\left[6^{1 / 2}\left(\frac{1}{7}\right) \hat{P}_{2}(\theta)\left(D_{0,-2}^{(2)}(\alpha, \beta, \gamma) m_{+1,+1}^{(0)}(\alpha, \beta, \gamma)+\text { c.c. }\right)\right. \\
& \left.+\left(\frac{2}{5}\right)^{1 / 2}\left(\frac{6}{7}\right) \hat{P}_{4}(\theta)\left(D_{0,-2}^{(4)}(\alpha, \beta, \gamma) m_{+1,+1}^{(0)}(\alpha, \beta, \gamma)+\text { c.c. }\right)\right] \\
& -4 \times 3^{1 / 2}\left[+\left(\frac{1}{7}\right) \hat{P}_{2}(\theta)\left(D_{0,-1}^{(2)}(\alpha, \beta, \gamma) m_{+1,0}^{(0)}(\alpha, \beta, \gamma)+\text { c.c. }\right)\right. \\
& \left.\quad+\left(\frac{6}{5}\right)^{1 / 2}\left(\frac{3}{7}\right) \hat{P}_{4}(\theta)\left(D_{0,-1}^{(4)}(\alpha, \beta, \gamma) m_{+1,0}^{(0)}(\alpha, \beta, \gamma)+\text { c.c. }\right)\right] .
\end{aligned}
$$

This expression is the counterpart of Eq. [40] and can be used, after spatial averaging, for the evaluation of $\left(T_{2 p}\right)_{\text {dip }}^{-1}$. Like $\tilde{\Theta}_{1}(\beta), \tilde{\Theta}_{2}(\beta)$ is a function only of the Euler angle $\beta$ (see above), and its spatial average can be calculated as in Eq. [41].

\section{BEHAVIOR IN THE ZEEMAN LIMIT}

Extrapolations of Eqs. [40] and [45] to the limiting forms of the Zeeman and zfs theories is reasonably straightforward. The correspondence of Eq. [40] with SBM theory (the Zeeman limit) will be illustrated first. In the Zeeman limit, $\mathscr{H}_{\mathrm{S}}^{(0)} \rightarrow$ $\mathscr{H}_{\mathrm{Z}}^{(0)}$; the eigenfunctions reduce to the Zeeman basis functions, $|\mu\rangle,|\nu\rangle \rightarrow|m\rangle$, $|n\rangle$; and the spectral density functions $m_{p, p^{\prime}}^{( \pm)}$can be written

$$
m_{p, \nu^{\prime}}^{( \pm)}=(2 S+1)^{-1} \sum_{m, n}\left\langle m\left|S_{p}^{(1)}\right| n\right\rangle\left\langle n\left|S_{p^{\prime}}^{(1)}\right| m\right\rangle j\left(\omega_{m}-\omega_{n} \pm \omega_{1}\right)
$$

The matrix elements $\left\langle m\left|S_{p}^{(1)}\right| n\right\rangle$ can be evaluated analytically,

$$
\begin{aligned}
\left\langle m\left|S_{0}^{(1)}\right| m\right\rangle & =m \\
\left\langle m \pm 1\left|S_{ \pm 1}^{(1)}\right| m\right\rangle & =\mp 2^{-1 / 2} c_{\mathrm{S}, m}^{ \pm},
\end{aligned}
$$

where $c_{\mathrm{S}, m}^{ \pm}$are the raising and lowering operators. All terms $m_{p, p}^{( \pm)}$, vanish except for those with $p+p^{\prime}=0$ and $m-n=0, \pm 1$. The spectral density functions $j\left(\omega_{m}-\omega_{n}\right.$ $\left.\pm \omega_{\mathrm{I}}\right)$ in the nonzero terms are $j\left(\omega_{\mathrm{S}}\right)$ for $m=n \pm 1$, and $j\left(\omega_{\mathrm{I}}\right)$ for $m=n$. This gives

$$
\begin{aligned}
& m_{0,0^{\prime}}^{( \pm)}=(2 S+1)^{-1} \sum_{m} m^{2} j\left(\omega_{\mathrm{I}}\right)=\frac{S(S+1)}{3} j\left(\omega_{\mathrm{I}}\right) \\
& m_{ \pm 1, \mp 1}^{( \pm)}=(2 S+1)^{-1} \sum_{m}(-2)^{-1}\left|c_{\mathrm{S}, m}^{\mp}\right|^{2} j\left(\omega_{\mathrm{S}}\right)=-\frac{S(S+1)}{3} j\left(\omega_{\mathrm{S}}\right),
\end{aligned}
$$


where the identities

$$
(2 S+1)^{-1} \sum_{m} m^{2}=2^{-1}(2 S+1)^{-1} \sum_{m}\left|c_{\mathrm{S}, m}^{ \pm}\right|^{2}=\frac{S(S+1)}{3}
$$

have been used. It is evident from Eqs. [48] that the functions $m_{p, p^{\prime}}^{( \pm)}$, are independent of molecular orientation in the Zeeman limit. (This fact is also evident on physical grounds, since the spin Hamiltonians of both $\mathbf{S}$ and $\mathbf{I}$ are Zeeman Hamiltonians and independent of the Euler angles.)

When these results are inserted in the expression for $\tilde{\Theta}_{1}(\beta)$ (Eq. [40]), the third and fourth terms vanish due to the restriction $p+p^{\prime}=0$ in the trace over spin variables (Eq. [46]). Furthermore, in the first and second terms of Eq. [40], the parts proportional to $P_{2}(\beta)$ and $P_{4}(\beta)$ vanish in the spatial average since $m_{p, p}^{( \pm)}$, is independent of the spatial variables and since $\left\{P_{n}(\beta)\right\}_{\text {sp av }}=0$ for all $n$ other than zero. This gives the very simple result that only the constant terms of Eq. [40] (i.e., those independent of $\beta$ ) are nonzero:

$$
\tilde{\Theta}_{1}=10^{-1}\left\{-\left(\frac{6}{5}\right) m_{0,0}^{(+)}+\left(\frac{14}{5}\right) m_{+1,-1}^{( \pm)}\right\} .
$$

Using Eqs. [48] for $m_{p, p}^{( \pm)}$, gives for $\tilde{\Theta}_{1}$

$$
\tilde{\Theta}_{1}=10^{-1}\left(\frac{2}{5}\right) \frac{S(S+1)}{3}\left\{-3 j\left(\omega_{\mathrm{I}}\right)-7 j\left(\omega_{\mathrm{S}}\right)\right\}
$$

and for $\left(T_{1 p}\right)_{\mathrm{dip}}^{-1}$

$$
\left(T_{1 p}\right)_{\mathrm{dip}}^{-1}=\left(\frac{g \beta_{0} B_{0}}{r^{3}}\right)^{2}\left(\frac{\mu_{0}}{4 \pi}\right)^{2}\left(\frac{2}{15}\right) S(S+1)\left\{3 j\left(\omega_{\mathrm{I}}\right)+7 j\left(\omega_{\mathrm{S}}\right)\right\} .
$$

This agrees with SBM theory in the approximation (which was invoked in the definition of the $\left.m_{p, p^{\prime}}^{( \pm)}\right)$that $j\left(\omega_{\mathrm{S}}\right) \approx j\left(\omega_{\mathrm{S}} \pm \omega_{\mathrm{I}}\right)$.

\section{THE zfs LIMIT}

As in the Zeeman limit, $\left(T_{1 p}\right)_{\text {dip }}^{-1}$ reduces in the zfs limit to a fairly simple analytical form. In this limit, the static electron spin Hamiltonian $\mathscr{H}_{\mathrm{S}}^{(0)}$ is the static zfs Hamiltonian $\mathscr{H}_{\mathrm{zfs}}^{(0)}$, with eigenfunctions $\left|\mu_{0}\right\rangle,\left|\nu_{0}\right\rangle$ and eigenvalues $\mu_{0}$ and $\nu_{0}$. As in the Zeeman limit, the functions $m_{p, p^{\prime}}^{( \pm)}$can be expressed analytically, although for this purpose it is first necessary to write the spin variables $S_{p}^{(1)}$ in the molecular coordinate system:

$$
S_{p}^{(1)}=\sum_{p^{\prime}} \hat{S}_{p^{\prime}}^{(1)} \mathcal{D}_{p^{\prime}, p}^{(1)}(\alpha, \beta, \gamma)
$$

Then the matrix elements $\left\langle\mu_{0}\left|\hat{S}_{p}^{(1)}\right| \nu_{0}\right\rangle$ can be evaluated as in Eqs. [47], giving for the $m_{p, p^{\prime}}^{( \pm)}$

$$
\begin{aligned}
& m_{0,0}^{( \pm)}=(2 S+1)^{-1} \sum_{\mu_{0}}\left\{\mu_{0}^{2} \mathscr{D}_{0,0}^{(1)} \mathscr{D}_{0,0}^{(1)} j\left(\omega_{1}\right)\right. \\
& \left.+(-2)^{-1}\left(\left|c_{\mathrm{S}, \mu_{0}}^{+}\right|^{2}+\left|c_{\mathrm{S}, \mu_{0}}^{-}\right|^{2}\right) \mathscr{D}_{-1,0}^{(1)} \mathcal{D}_{+1,0}^{(1)} j\left(\omega_{\mu_{0}}\right)\right\} \\
& m_{ \pm 1, \mp 1}^{( \pm)}=(2 S+1)^{-1} \sum_{\mu_{0}}\left\{\mu_{0}^{2} \mathscr{D}_{0, \pm 1}^{(1)} \mathcal{D}_{0, \mp 1}^{(1)} j\left(\omega_{\mathrm{I}}\right)\right. \\
& \left.+(-2)^{-1}\left|c_{\mathrm{S}, \mu_{0}}^{+}\right|^{2}\left(\mathcal{D}_{-1,+1}^{(1)} \mathcal{D}_{+1-1}^{(1)}+\mathscr{D}_{-1,-1}^{(1)} \mathcal{D}_{+1,+1}^{(1)}\right) j\left(\omega_{\mu_{0}}\right)\right\}
\end{aligned}
$$


with $m_{p, p^{\prime}}^{( \pm)}=0$ when $p+p^{\prime} \neq 0$. The pairwise products can then be contracted using the Clebsch-Gordan relation (Eq. [23]), giving

$$
\begin{aligned}
m_{0,0}^{( \pm)}=\frac{S(S+1)}{3} 3^{-1}\left(1+2 D_{0,0}^{(2)}\right) j\left(\omega_{\mathrm{I}}\right) & \\
& +\left(-3^{-1}\right)\left(-1+\mathscr{D}_{0,0}^{(2)}\right) \sum_{\mu_{0}}\left|c_{\mathrm{S}, \mu_{0}}^{+}\right|^{2} j\left(\omega_{\mu_{0}}\right), \\
m_{ \pm 1, \mp 1}^{( \pm)}=\frac{S(S+1)}{3} 3^{-1}\left(-1+\mathcal{D}_{0,0}^{(2)}\right) j\left(\omega_{1}\right) & \\
& +3^{-1}\left(1+2^{-1} \mathscr{D}_{0,0}^{(2)}\right) \sum_{\mu_{0}}\left|c_{\mathrm{S}, \mu_{0}}^{+}\right|^{2} j\left(\omega_{\mu_{0}}\right) .
\end{aligned}
$$

Equations [55a] and [55b] display the explicit spatial dependence of the $m_{p, p}^{( \pm)}$, in simplest form. These expressions can be inserted into Eq. [41] and the required spatial averages calculated analytically. For this purpose, it is convenient to use the following formulas for the spatial averages of the rotation-matrix elements:

$$
\left\{D_{q_{1}, q_{2}}^{(j)}\right\}_{\text {sp av }}=0
$$

unless $q_{1}=q_{2}=j=0$, in which case the average equals unity, and, for the pairwise products,

$$
\left\{\mathcal{D}_{q_{1}, q_{1}^{\prime}}^{\left(j_{1}\right)}, \mathscr{D}_{q_{2}, q_{2}}^{\left(j_{2}\right)}\right\}^{\text {sp av }}=\left(2 j_{1}+1\right)^{-1} \delta_{q_{1} q_{2}} \delta_{q_{1}^{\prime} q_{2}^{\prime}} \delta_{j_{1} j_{2}} .
$$

Due to the restrictions on the indices $p, p^{\prime}$ in the matrix elements of $\mathbf{S}$ and on the indices $\left(q_{1}, q_{1}^{\prime}\right),\left(q_{2}, q_{2}^{\prime}\right)$ and $\left(j_{1}, j_{2}\right)$ that result from the spatial average, the nonvanishing portion of $\tilde{\Theta}_{1}$ in the zfs limit reduces to

$$
\tilde{\Theta}_{1}=10^{-1}\left\{6 m_{0,0}^{( \pm)}\left(-5^{-1}-7^{-1} \hat{P}_{2} D_{0,0}^{(2)}\right)+2 m_{+1,-1}^{( \pm)}\left(\frac{7}{5}-\frac{10}{7} \hat{P}_{2} D_{0,0}^{(2)}\right)\right\} .
$$

Using Eqs. [41] and [54]-[58], the zfs-limit expression for $\left(T_{1 p}\right)_{\text {dip }}^{-1}$ is

$$
\begin{aligned}
\left(T_{1 p}\right)_{\text {dip }}^{-1}=\frac{4}{3}\left(\frac{\gamma_{1} g_{0} \beta_{0}}{r^{3}}\right)^{2}\left(\frac{\mu_{0}}{4 \pi}\right)^{2} & \left\{\frac{S(S+1)}{3}\left[1+\hat{P}_{2}(\theta)\right] j\left(\omega_{\mathrm{I}}\right)\right. \\
& \left.+(2 S+1)^{-1}\left[1-2^{-1} \hat{P}_{2}(\theta)\right] \sum_{\mu_{0}}\left|c_{\mathrm{S}, \mu_{0}}^{+}\right|^{2} j\left(\omega_{\mu_{0}}\right)\right\} .
\end{aligned}
$$

The corresponding result for $\left(T_{2 p}\right)_{\mathrm{dip}}^{-1}$ is identical to Eq. [59] except for the replacement of $j\left(\omega_{\mathrm{I}}\right)$ by $j(0)$. The present result (Eq. [59]) is significantly simpler in mathematical form than the corresponding result published previously (5). By applying tensor contraction methods, the earlier result (Eq. [19] in Ref. (5)) can be shown to be equivalent mathematically to Eq. [59] above.

\section{COMPARISON WITH DATA FOR $\mathrm{Mn}(\mathrm{AcAc})_{3}$}

$\mathrm{Mn}$ (III)(AcAc) $)_{3}$ contains a high-spin $\mathrm{Mn}$ (III) ion $\left(d^{4}, S=2\right)$. This complex is well-defined structurally and with respect to its electron and nuclear-spin relaxation and static magnetic susceptibility properties and provides a suitable system for examining quantitatively the theory developed above. 
$\mathrm{Mn}$ (III)(AcAc) $)_{3}$ has overall $D_{3}$ symmetry and is shown schematically in Fig. 1 in a view down the $D_{3}$ axis. The methyl groups of the ligands are shown as circles, three of which (filled circles) lie above the plane of the $C_{2}$ axes, and three of which (unfilled circles) lie below this plane. The $\mathrm{Mn}$ (III) ion is coordinated octahedrally by six oxygens, none of which coincides with a symmetry element of the $D_{3}$ point group. The apparent $D_{3}$ symmetry is broken down by a Jahn-Teller distortion along one of the coordination axes. The crystal structure of $\mathrm{Mn}(\mathrm{AcAc})_{3}$ exhibits two long (2.11 $\AA$ ) and four short ( $1.94 \AA$ ) $\mathrm{Mn}-\mathrm{O}$ bonds (26). This structure indicates that the zfs tensor of $\mathrm{Mn}$ (III) is approximately uniaxial, with its unique axis aligned with the octahedral coordination sphere. This direction is indicated by the arrow in Fig. 1. Static magnetic susceptibility measurements (20) have given a value of $D=-3.1 \mathrm{~cm}^{-1}$ for the zfs parameter. The electron-spin relaxation properties of $\mathrm{Mn}(\mathrm{III})(\mathrm{AcAc})_{3}$ have also been studied (5).

Intramolecular $R_{1 p}$ data for the methyl protons of $\mathrm{Mn}(\mathrm{AcAc})_{3}$ have been reported as a function of field strength in the range $0.214-6.53 \mathrm{~T}(22) . R_{1 p}$ is nearly constant across this range, decreasing by about $20 \%$ at the high-field end. These data are shown in Fig. 3 along with theoretical curves of $R_{1 p}$ vs $B_{0}$, calculated (1) using SBM theory and (2) with the theory developed above by averaging Eq. [40] in accord with Eq. [51]. Physical parameters for the calculations were taken to be $r_{\mathrm{I}-\mathrm{S}}=5.0 \AA$ (estimated from molecular models), the zero-field splitting parameter $D=-3.1 \mathrm{~cm}^{-1}$, and the magnetic field strength $B_{0}=0.214 \mathrm{~T}$. For the electron-spin relaxation time $\tau_{\mathrm{S}}$, the values previously measured (5) from solvent proton relaxation enhancement data were used, $\tau_{\mathrm{S}}=8 \mathrm{ps}$ at the $\mathrm{zfs}$ limit and $\tau_{\mathrm{S}}=2.3 \mathrm{ps}$ at the Zeeman limit.

Outside the Zeeman limit, the calculated $R_{1 p}$ is a function of the polar angle $\hat{\theta}$ of the methyl protons in the molecular frame. An accurate specification of $\hat{\theta}$ for each of the 12 methyl protons is not simple due to effects of internal degrees of freedom, both fluctional and those pertaining to rotamer populations, which are not known with high accuracy. From Fig. 1 it can be seen that two methyls lie near the $\hat{z}$ axis, while the other four are nearer the $\hat{x} \hat{y}$ plane. An effective value of $\hat{\theta}$ is roughly one radian.

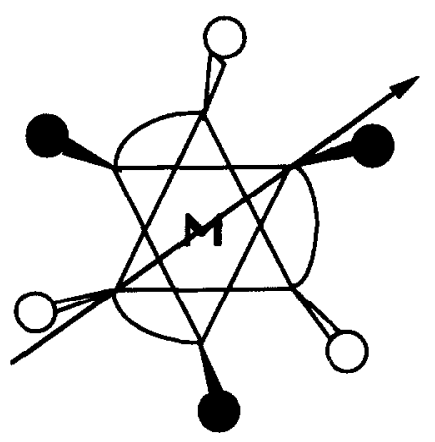

FIG. 1. Schematic representation of tris(acetylacetonato) metal(III) complexes. View down the $D_{3}$ symmetry axis of the complex. Methyl groups are indicated as circles. Solid circles are methyls projecting above the median plane of the complex, and open circles are methyls below the median plane. The Jahn-Teller distortion [when present, as in the Mn(III) complex ] occurs along a fourfold axis of the metal coordination sphere as indicated by the arrow. 
To provide an idea of the dependence of the calculation on the assumed molecular geometry, $R_{1 p}$ was computed as a function of $\hat{\theta}$ (Fig. 2 ). Arrows in the figure correspond approximately to $\hat{\theta}$ values of the 6 methyl carbons. The measured $R_{1 p}$ value at 0.214 $T$ is indicated by the horizontal line. Considering that the calculation contains no adjustable parameters other than $\hat{\theta}$ ( $\tau_{\mathrm{S}}$ was measured independently), the quantitative agreement between theory and experiment is quite good.

The magnetic field dependence of $R_{1 p}$ was then calculated and compared with experimental data (Fig. 3). The SBM (Zeeman-limit) calculations were carried out following Refs. $(4,30,31)$. In the Zeeman limit, $\tau_{\mathrm{S}}$ is in general field-dependent as given by (30)

$$
\left(\tau_{\mathrm{S}}\right)^{-1}=\left(\tau_{\mathrm{S}}^{(0)}\right)^{-1}\left\{\frac{0.2}{1+\omega_{\mathrm{S}}^{2} \tau_{\mathrm{v}}^{2}}+\frac{0.8}{1+4 \omega_{\mathrm{S}}^{2} \tau_{\mathrm{v}}^{2}}\right\},
$$

where $\tau_{\mathrm{S}}^{(0)}$ is the low-field limit value of $\tau_{\mathrm{S}}, \omega_{\mathrm{S}}$ is the electronic Larmor frequency, and $\tau_{\mathrm{v}}$ is the damping time of asymmetric vibrational modes of the $\mathrm{Mn}$ (III) coordination sphere. The measured Zeeman-limit value of $\tau_{\mathrm{S}}, \tau_{\mathrm{S}}=2.3 \mathrm{ps}$ at $12.63 \mathrm{MHz}$ (5), was taken as $\tau_{S}^{(0)} ; \tau_{v}$ was taken to be $5 \mathrm{ps,} \mathrm{the} \mathrm{measured} \mathrm{value} \mathrm{for} \operatorname{Mn}$ (II) (30). The value of $\tau_{\mathrm{S}}$ exhibits very strong field dependence above about $1 \mathrm{~T}$, and this fact is mirrored in the behavior of $R_{1 p}$. Overall, the data are not at all well described by SBM theory.

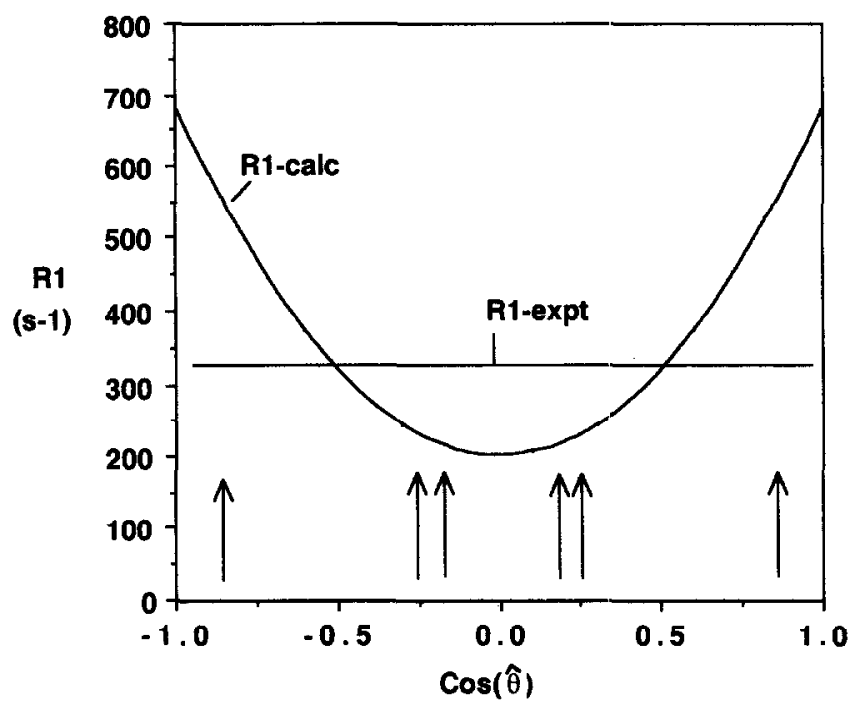

FIG. 2. Calculated dipolar paramagnetic relaxation rate $R_{1_{p}}$ of the methyl protons of tris(acetylacetonato) $\mathrm{Mn}$ (III) as a function of the polar angle $(\hat{\theta})$ of the interspin I-S vector in the molecular coordinate frame that diagonalizes the zfs tensor. The calculation assumes as I-S distance $r_{\mathrm{I}-\mathrm{s}}=5.0 \AA$, a $\mathrm{zfs}$ parameter $|D|=3.1 \mathrm{~cm}^{-1}$, a field strength $B_{0}=0.214 \mathrm{~T}$, and an electron-spin relaxation time $\tau_{\mathrm{S}}=8.0 \mathrm{ps}$. The experimental $R_{1_{p}}$ measured at $0.214 \mathrm{~T}$ and room temperature (22), is also shown. Arrows indicate, approximately, the orientations of the six methyl groups in the molecular coordinate system, for which the $\hat{z}$ axis lies along a fourfold axis of the manganese coordination sphere. 


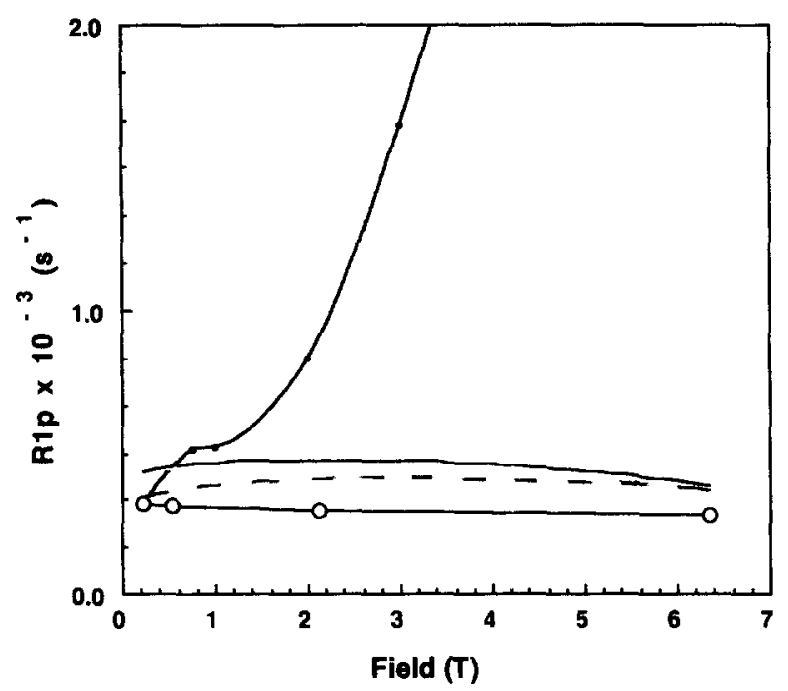

FIG. 3. Magnetic field dependence of the $T_{\mathfrak{l}}$ relaxation rate of the methyl proton resonance in tris(acetylacetonato) $\mathrm{Mn}$ (III) in solution (room temperature). Open circles are data from Ref. (22). Solid and dashed curves were calculated using Eq. [40] with the following parameters: $r_{1-\mathrm{s}}=5.0 \AA$, $\tau_{\mathrm{S}}=8.0 \mathrm{ps}$, zfs parameter $D=-3.1 \mathrm{~cm}^{-1}$. The average value of $\hat{\theta}$, the polar angle of the I-S vector in the molecular frame, was set at 0.8 (solid curve) and $1.0 \mathrm{rad}$ (dashed curve). Zeeman limit calculations (dashed curve) were carried out as in Refs. $(30,3 I)$ and utilized the following parameters: $r_{\mathrm{I}-\mathrm{S}}=5.0 \AA, \tau_{\mathrm{S}}^{(0)}=2.3 \mathrm{ps}$, and $\tau_{\mathrm{v}}=5$ ps.

Calculations based on Eqs. [40] and [41] are also shown in Fig. 3. The zero-field value of $\tau_{\mathrm{S}}$ derived from zfs-limit theory is $8 \mathrm{ps}(5)$. In the zfs limit, $\tau_{\mathrm{S}}$ is field-independent since the electronic energy-level spacings are independent of magnetic field strength. Field-independent behavior in $\tau_{\mathbf{S}}$ has been confirmed experimentally at field strengths between 0.1 and $1.9 \mathrm{~T}$, and in the calculation this behavior was assumed to extend out to $6.53 \mathrm{~T}$. Two sets of calculations, based on assumed $\hat{\theta}$ values of 0.8 and $1.0 \mathrm{rad}$, are illustrated in Fig. 6 . The calculations with $\hat{\theta}=0.8$ quite accurately predict the field dependence, including the observed mild drop in $R_{1 p}$. The absolute difference between theory and experiment is generally consistent with the uncertainties in the assumed value of $r_{1-S}$ and in the measured value of $\tau_{\mathrm{S}}$.

\section{CONCLUSIONS}

The theory developed above describes the dipolar portion of spin relaxation rates of solvent nuclei in solutions that contain dissolved paramagnetic metal ions with $S$ $>\frac{1}{2}$. It is intended to provide a bridge between the Zeeman- and zfs-limit expressions in Refs. $(1,2,5,6)$. The physical assumptions employed are appropriate to situations where the electronic and Zeeman energies are of arbitrary magnitude, where the $\mathrm{zfs}$ tensor possesses uniaxial symmetry, and where the correlation times for molecular reorientation are slow on the time scale of electron-spin relaxation.

The electron-spin relaxation time has been treated as a fixed parameter without any explicit incorporation of its magnetic field dependence. In the Zeeman limit, $\tau_{\mathbf{S}}$ 
is often field-depenedent with a behavior that depends critically on the spacing and field dependence of the electron-spin energy levels. It should be emphasized here that it is not appropriate to "extend" the present theory [or the zfs-limit expressions (5, $6)]$ through the use of a Zeeman-limit theory for $\tau_{\mathrm{S}}$, such as that of Bloembergen and Morgan (3). Pegg et al. (22) have pointed out that in the zfs limit, the field dependence of $\tau_{\mathrm{S}}$ is expected be small because the spin energy level spacings are to first order independent of the Zeeman interaction. This situation differs for integer and halfinteger spins, however, since the $\pm \frac{1}{2}$ levels of half-integer spins exhibit Zeeman behavior (with a fictitious spin quantum number) is lowest order. For integer spins, a simple but reasonable assumption is that $\tau_{\mathrm{S}}$ is approximately field-independent when the Zeeman energy is smaller than the $\mathrm{zfs}$ energy and when the spin system is not near an energy level crossing. This assumption was made implicitly above in the analysis of $\mathrm{Mn}(\mathrm{AcAc})_{3}$ data, where there is clear experimental evidence (5) for its validity, at least in the range of field strengths below $2 \mathrm{~T}$.

\section{COMPUTER PROGRAM "PARELAX"}

To facilitate practical applications, a computer program, "Parelax," has been written using the high-level programming language Mathematica for the MacIntosh II computer. This program handles intramolecular $T_{1}$ and $T_{2}$ relaxation arising from electronic spins of $S=1, \frac{3}{2}, 2$, and $\frac{5}{2}$ and can be supplied upon request.

\section{APPENDIX A}

\section{Restrictions on the Indices of Nonvanishing Terms in Eqs. [21] and [42]}

Eqs. [21] and [42] simplify considerably due to the fact that the majority of the tensor products vanish during the average over molecular orientations and in the trace over spin variables. This section examines restrictions resulting from the spatial average.

The Euler angles which describe the molecular orientation in the laboratory frame occur explicitly in the Wigner rotation-matrix elements $\mathscr{D}_{q,+1-p}^{(2)}(\alpha, \beta, \gamma) \mathcal{D}_{q,-1-p^{\prime}}^{(2)}(\alpha$, $\beta, \gamma)$ in Eqs. [21] and [42]. They also occur implicitly in the spin products

$$
M_{p, p^{\prime}}(t)=\exp \left(i \mathscr{H}_{\mathrm{S}}^{(0)} t\right) S_{p}^{(1)} \exp \left(-i \mathscr{H}_{\mathrm{S}}^{(0)} t\right) S_{p^{\prime}}^{(1)} \exp \left(-t / \tau_{\mathrm{S}}\right),
$$

due to the dependence of $\mathscr{H}_{\mathrm{S}}^{(0)}$ on molecular orientation as given by Eq. [30]. Writing the time evolution operator as

$$
\exp \left( \pm i \mathscr{H}_{\mathrm{S}}^{(0)} t\right)=\sum_{n=0}^{\infty} \frac{\left( \pm i \mathscr{H}_{\mathrm{S}}^{(0)} t\right)^{n}}{n !}
$$

and then expanding the resulting products give an infinite series in which the dependence on molecular orientation is exhibited explicitly as a product of Wigner rotation matrices in each term.

While the direct evaluation of this infinite series is certainly intractable in detail, an important restriction on nonvanishing terms can be demonstrated by contracting each product of rotation-matrix elements, $\mathscr{D}_{m_{1}, m_{1}^{\prime}}^{\left(j_{1}\right)}(\alpha, \beta, \gamma) \mathcal{D}_{m_{2}, m_{2}^{\prime}}^{\left(j_{2}\right)}(\alpha, \beta, \gamma), \ldots$, to a sum of terms, each of which contains a single rotation-matrix element. From the Clebsch-Gordan series (Eq. [23]), the pairwise product of any two rotation-matrix 
elements can be contracted to a sum of single rotation-matrix elements. According to the properties of the $3-j$ symbols, the pairwise product vanishes except for terms with $m=m_{1}+m_{2}$ and $m^{\prime}=m_{1}^{\prime}+m_{2}^{\prime}$. Thus, the sums of the orders on the 1.h.s. of Eq. [23] are strictly conserved in the orders $\left(m, m^{\prime}\right)$ on the r.h.s. of this equation. Similarly, any general product of rotation-matrix elements can be reduced to a sum of terms, each containing a single rotation-matrix element, the orders $\left(m_{1}, m_{2}\right)$ of which are equal to the sums of orders of all factors in the product,

$$
\prod_{i} D_{m_{1}, m_{2}^{(i)}}^{\left(j_{i}\right)}=\sum_{j} \mathbb{F}_{j} \mathcal{D}_{m_{1}, m_{2}}^{(i)}
$$

where $m_{1}=\Sigma_{i} m_{1}^{(i)}, m_{2}=\Sigma_{i} m_{2}^{(i)}, j$ satisfies the "triangle" relationship for the addition of angular momenta, $\Delta\left(j_{1} j_{2} \cdots\right)$ and the $\mathbb{F}_{j}$ are numerical factors.

Similarly, any general term $\pi$ in Eq. [21] can be written

where

$$
\begin{aligned}
& \mathbb{T}=g(t) f\left(S_{\rho}^{(j)}\right) \mathcal{D}_{q,+1-p}^{(2)} \mathcal{D}_{q^{\prime},+1-p^{\prime}}^{(2)} \prod_{i} \mathcal{D}_{0,-\lambda_{i}}^{(2)} \prod_{i^{\prime}} \mathcal{D}_{0,-\lambda_{i^{\prime}}}^{(2)} \\
& \mathbb{T}=g(t) f\left(S_{\rho}^{(j)}\right) \sum_{j} \mathscr{D}_{q^{+} q^{\prime}, \sigma}^{(j)}(\alpha, \beta, \gamma),
\end{aligned}
$$

$$
\sigma=+2-p-p^{\prime}-\sum_{i} \lambda_{i}-\sum_{i^{\prime}} \lambda_{i^{\prime}}
$$

Averaging $\mathbb{T}$ in a termwise manner over molecular orientations, i.e., over the space of the Euler angles, then involves the average of only a single rotation-matrix elcment in each term. In an isotropic environment, only $D_{0,0}^{(0)}(\alpha, \beta, \gamma)$ (i.e., the term with $j=$ $0, q+q^{\prime}=0$, and $\sigma=0$ ) gives a nonvanishing spatial average. This leads immediately to the restriction $q^{\prime}=-q$. There is no such restriction on the indices $p, p^{\prime}$.

\section{APPENDIX B}

Formulas for Contraction of Sums of Tensor Products of the Form

$$
\Sigma_{q} \hat{C}_{q}^{(2)}(\theta, \varphi) \hat{C}_{-q}^{(2)}(\theta, \varphi) D_{q, r}^{(2)}(\alpha, \beta, \gamma) \mathcal{D}_{-q, s}^{(2)}(\alpha, \beta, \gamma)
$$

Sums of this form can be reduced, following application of the Clebsch-Gordan formula (Eq. [23]), to a sum of three terms of the form

$$
\sum_{q} \hat{C}_{q}^{(2)} \hat{C}_{-q}^{(2)} \mathcal{D}_{q, r}^{(2)} \mathcal{D}_{-q, s}^{(2)}=A+B \hat{P}_{2}(\theta) \mathcal{D}_{0, r+s}^{(2)}(\beta)+C \hat{P}_{4}(\theta) \mathcal{D}_{0, r+s}^{(4)}(\beta)
$$

The numerical coefficients $A, B$, and $C$ are tabulated below. The reduction of these sums can be accomplished in the following steps:

(1) Each pairwise product of rotation-matrix elements on the 1.h.s. of [B1] is contracted to a sum of terms using the Clebsch-Gordan relation (Eq. [23]); e.g., for the term with $q=0, r=+1, s=-1$,

$$
\mathscr{D}_{0,+1}^{(2)} \mathscr{D}_{0,-1}^{(2)}=-5^{-1} \mathscr{D}_{0,0}^{(0)}-7^{-1} \mathscr{D}_{0,0}^{(2)}+2^{2} 3^{1} 5^{-1} 7^{-1} \mathscr{D}_{0,0}^{(4)}
$$

(2) Terms in the sum over the index $q$ were collected in a similar manner, collecting as factors the coefficients of each rotation-matrix element of the contracted sum, e.g., 


$$
\begin{aligned}
& \sum_{q} \hat{C}_{q}^{(2)} \hat{C}_{-q}^{(2)} D_{q,+1}^{(2)} D_{-q,-1}^{(2)}=-5^{-1}\left(\hat{C}_{0}^{(2)} \hat{C}_{0}^{(2)}-\hat{C}_{+1}^{(2)} \hat{C}_{-1}^{(2)}-2 \hat{C}_{+2}^{(2)} \hat{C}_{-2}^{(2)}\right) D_{0,0}^{(0)} \\
&-7^{-1}\left(-\hat{C}_{0}^{(2)} \hat{C}_{0}^{(2)}+\hat{C}_{+1}^{(2)} \hat{C}_{-1}^{(2)}-2 \hat{C}_{+2}^{(2)} \hat{C}_{-2}^{(2)}\right) D_{0,0}^{(2)} \\
&+2^{2} 5^{-1} 7^{-1}\left(\hat{C}_{0}^{(2)} \hat{C}_{0}^{(2)}+\frac{4}{3} \hat{C}_{+1}^{(2)} \hat{C}_{-1}^{(2)}+\frac{1}{3} \hat{C}_{+2}^{(2)} \hat{C}_{-2}^{(2)}\right) \mathcal{D}_{0,0}^{(4)}
\end{aligned}
$$

(3) In every case, the Racah spherical harmonics can be grouped into coefficients of the rotation-matrix elements with the forms

$$
\begin{aligned}
\text { of } \mathcal{D}_{0,0}^{(0)}: & \hat{P}_{0} \equiv 1=\hat{C}_{0}^{(2)} \hat{C}_{0}^{(2)}-\hat{C}_{+1}^{(2)} \hat{C}_{-1}^{(2)}-2 \hat{C}_{+2}^{(2)} \hat{C}_{-2}^{(2)} \\
\text { of } D_{0,0}^{(2)}: & \hat{P}_{2}=-\hat{C}_{0}^{(2)} \hat{C}_{0}^{(2)}+\hat{C}_{+1}^{(2)} \hat{C}_{-1}^{(2)}-2 \hat{C}_{+2}^{(2)} \hat{C}_{-2}^{(2)} \\
\text { of } D_{0,0}^{(4)}: & \hat{P}_{4}=+\hat{C}_{0}^{(2)} \hat{C}_{0}^{(2)}+\frac{4}{3} \hat{C}_{+1}^{(2)} \hat{C}_{-1}^{(2)}+\frac{1}{3} \hat{C}_{+2}^{(2)} \hat{C}_{-2}^{(2)} .
\end{aligned}
$$

The $\hat{P}_{n}$ are Legendre polynomials of order $n$ in $\cos \theta$. (The identities [B4a]- [B4c] can be confirmed by contracting each of the pairwise products $\hat{C}_{+q}^{(2)} \hat{C}_{-q}^{(2)}$ with Eq. [23] and using the relation $\left.\hat{P}_{2}(\theta)=\hat{C}_{0}^{(2)}(\theta, \varphi)\right)$.

(4) With the use of Eqs. [B4a]-[B4c], sums of the form [B1] can be constructed. Coefficients $A, B$, and $C$ are tabulated below as a function of $r$ and $s$. Each of the relations below has been confirmed numerically using randomly selected values of the angular variables.

\begin{tabular}{rrrll}
$r$ & $s$ & $A$ & \multicolumn{1}{c}{$B$} & \multicolumn{1}{c}{$C$} \\
\hline 0 & 0 & $5^{-1}$ & $2^{1} 7^{-1}$ & $2^{1} 3^{2} 5^{-1} 7^{-1}$ \\
+1 & -1 & $-5^{-1}$ & $-7^{-1}$ & $2^{2} 3^{1} 5^{-1} 7^{-1}$ \\
+2 & -2 & $5^{-1}$ & $-2^{1} 7^{-1}$ & $3^{1} 5^{-1} 7^{-1}$ \\
\pm 1 & 0 & 0 & $\pm 7^{-1}$ & $\pm 2^{1 / 2} 3^{3 / 2} 5^{-1 / 2} 7^{-1}$ \\
\pm 2 & 0 & 0 & $-2^{1} 7^{-1}$ & $3^{3 / 2} 5^{-1 / 2} 7^{-1}$ \\
\pm 1 & \pm 1 & 0 & $2^{1 / 2} 3^{1 / 2} 7^{-1}$ & $2^{3 / 2} 3^{1} 5^{-1 / 2} 7^{-1}$ \\
\pm 2 & \pm 2 & 0 & $2^{1 / 2} 3^{1 / 2} 7^{-1}$ & $2^{3 / 2} 3^{1} 5^{-1 / 2} 7^{-1}$
\end{tabular}

\section{APPENDIX C}

\section{Calculation of the Spatial Average of $\tilde{\Theta}_{1}(\alpha, \beta, \gamma)$}

We show in this section that integration over the space of the Euler angles $\alpha$ and $\gamma$ is not required in the evaluation of the spatial average of Eq. [40]. This fact, which is not particularly obvious from the form of Eq. [40], will be illustrated for one term of $\tilde{\Theta}_{1}$, namely the term containing the product $D_{0,-1}^{(2)}(\alpha, \beta, \gamma) m_{+1,0}^{( \pm)}(\alpha, \beta, \gamma)$. A straightforward extension of the reasoning should suffice to show that each term in Eq. [40] is a function of $\beta$ only.

The Wigner rotation-matrix elements can be written generally in the form

$$
\mathcal{D}_{q^{\prime}, q}^{(j)}(\alpha, \beta, \gamma)=e^{i \gamma q^{\prime}} d_{q^{\prime}, q}^{(j)}(\beta) e^{i \alpha q},
$$

in which the functional dependence on $\alpha$ and $\gamma$ is exhibited explicitly.

The angular dependence of the functions 


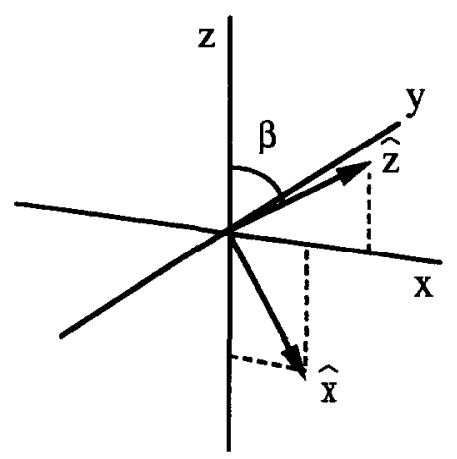

FIG. 4. Rotation of laboratory to molecular coordinate axes corresponding to the Euler angles $(\alpha, \beta, \gamma)$ $=(0, \beta, 0)$.

$$
m_{r, s}^{( \pm)}(\alpha, \beta, \gamma)=\sum_{\mu, \nu}\left\langle\mu\left|S_{r}^{(1)}\right| \nu\right\rangle\left\langle\nu\left|S_{s}^{(1)}\right| \mu\right\rangle j\left(\omega_{\mu}-\omega_{\nu}+\omega_{\mathrm{I}}\right)
$$

can be written in a similarly explicit form. For the sake of concreteness, the specific function $m_{+10}^{( \pm)}(\alpha, \beta, \gamma)$ will be considered. From physical symmetry, the eigenvalues $\omega_{\mu}, \omega_{\nu}$ of $\mathscr{H}_{\mathrm{S}}^{(0)}$, and hence also the spectral density functions, $j\left(\omega_{\mu}-\omega_{\nu} \pm \omega_{\mathrm{I}}\right)$, depend only on $\beta$ when the zfs tensor possesses uniaxial symmetry. The same is not true of the matrix elements, $\left\langle\mu\left|S_{q}^{(1)}\right| \nu\right\rangle$, however, which depend on the choice of laboratory $x$ and $y$ axes. To consider the transformation properties of $m_{+1,0}^{( \pm)}(\alpha, \beta, \gamma)$ with respect to $\alpha$ and $\gamma$ at fixed $\beta$, we consider first the molecular orientation in which the molecular $\hat{z}$ axis is at the polar angle $\beta$, with the laboratory $x$ axis in the $z, \hat{z}$ plane (Fig. 4). The Euler angles which transform the laboratory axes to the molecular axes in this case are $(0, \beta, 0)$, and the corresponding function $m_{+1,0}^{( \pm)}(\alpha, \beta, \gamma)$ is $m_{+1,0}^{( \pm)}(0, \beta, 0)$.

Next we consider the function $m_{+1,0}^{( \pm)}(\alpha, \beta, 0)$, which corresponds to a molecular orientation in which the $\tilde{z}$ axis is likewise at an angle $\beta$ with respect to the laboratory $z$ axis, but where the molecular axes have been rotated by an initial angle $\alpha$ about the

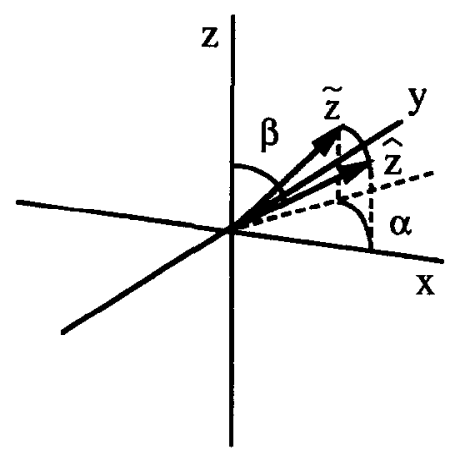

FIG. 5. Definition of the molecular $\hat{z}$ and $\tilde{z}$ axes corresponding to rotations of the laboratory axes through rutations by Euler angles of $(0, \beta, 0)$ and $(\alpha, \beta, 0)$, respectively. 
$z$ axis prior to the rotation by angle $\beta$ about the new $y$ axis ( $\tilde{y}$ ) (Fig. 5). Now the laboratory $x$ axis no longer lies in the $z, \tilde{z}$ plane (Fig. 6). A new function

$$
\tilde{m}_{+1,0}^{( \pm)}(0, \beta, 0)=e^{+i \omega_{I} t} \operatorname{Tr}\left\{\tilde{S}_{+1}^{(1)}(t) \tilde{S}_{0}^{(1)}(0)\right\}
$$

can be defined in terms of spin operators in the $(\tilde{x}, \tilde{y}, \tilde{z})$ coordinate system, which is related to the $(x, y, z)$ system by a rotation of angle $\alpha$ about $z$. Clearly

$$
\tilde{m}_{+1,0}^{( \pm)}(0, \beta, 0)=m_{+1,0}^{( \pm)}(0, \beta, 0)
$$

because of the axial physical symmetry in the problem. Now the desired function $m_{+1,0}^{( \pm)}(\alpha, \beta, 0)$ can be written in terms of the function $m_{+1,0}^{( \pm)}(0, \beta, 0)$ by writing the operators $S_{+1}^{(1)}=S_{0}^{(1)}$ in terms of those in the $(\tilde{x}, \tilde{y}, \tilde{z})$ system:

$$
\begin{aligned}
& S_{+1}^{(1)}=\tilde{S}_{+1}^{(1)} D_{+1,+1}^{(1)}(\alpha, 0,0,)+\tilde{S}_{0}^{(1)} \mathcal{D}_{0,+1}^{(1)}(\alpha, 0,0)+\tilde{S}_{-1}^{(1)} \mathscr{D}_{-1,+1}^{(1)}(\alpha, 0,0) \\
& S_{0}^{(1)}=\tilde{S}_{+1}^{(1)} \mathcal{D}_{+1,0}^{(1)}(\alpha, 0,0)+\tilde{S}_{0}^{(1)} \mathcal{D}_{0,0}^{(1)}(\alpha, 0,0)+\tilde{S}_{-1}^{(1)} \mathscr{D}_{-1,0}^{(1)}(\alpha, 0,0) .
\end{aligned}
$$

The specific forms for the rotation matrix elements $D_{q,+1}^{(1)}(\alpha, 0,0)$ and $\mathscr{D}_{q^{\prime}, 0}^{(1)}(\alpha, 0,0)$ in these expressions are

$$
\begin{gathered}
D_{+1,+1}^{(1)}(\alpha, 0,0)=e^{i \alpha} \\
D_{0,0}^{(1)}(\alpha, 0,0)=1 \\
D_{0,+1}^{(1)}(\alpha, 0,0)=D_{-1,+1}^{(1)}(\alpha, 0,0)=D_{+1,0}^{(1)}(\alpha, 0,0)=D_{-1,0}^{(1)}(\alpha, 0,0)=0 .
\end{gathered}
$$

The insertion of Eqs. [C5] into Eq. [C2] gives

$$
\begin{aligned}
m_{++1,0}^{( \pm)}(\alpha, \beta, 0)= & \sum_{\mu, \nu}\left[\sum_{q, q^{\prime}}\left\langle\mu\left|\tilde{S}_{q}^{(1)}\right| \nu\right\rangle\right. \\
& \left.\left\langle\nu\left|\tilde{S}_{q^{\prime}}^{(1)}\right| \mu\right\rangle D_{q,+1}^{(1)}(\alpha, 0,0) D_{q^{\prime}, 0}^{(1)}(\alpha, 0,0)\right] j\left(\omega_{\mu}-\omega_{\nu}+\omega_{I}\right),
\end{aligned}
$$

and, using the specific forms of the rotation-matrix elements (Eqs. [C6]), this becomes

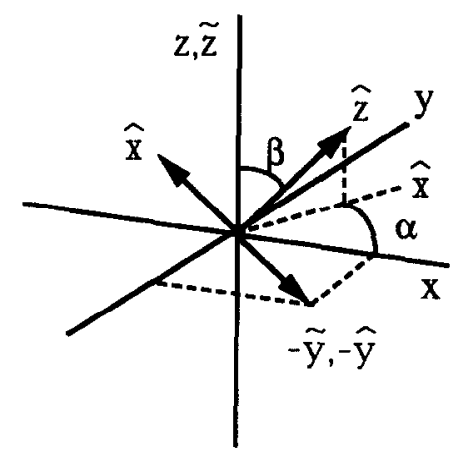

FIG. 6. Rotation of laboratory $(x, y, z)$ to molecular $(\tilde{x}, \tilde{y}, \tilde{z})$ coordinate axes corresponding to the Euler angles $(\alpha, \beta, \gamma)=(\alpha, \beta, 0)$. 


$$
\begin{aligned}
m_{+1,0}^{( \pm)}(\alpha, \beta, 0) & =e^{i \alpha} \sum_{\mu, \nu}\left\langle\mu\left|\tilde{S}_{+1}^{(1)}\right| \nu\right\rangle\left\langle\nu\left|\tilde{S}_{0}^{(1)}\right| \mu\right\rangle j\left(\omega_{\mu}-\omega_{\nu}+\omega_{\mathrm{I}}\right) \\
& =e^{i \alpha} \tilde{m}_{+1,0}^{( \pm)}(0, \beta, 0) \\
& =e^{i \alpha} m_{+1,0}^{( \pm)}(0, \beta, 0) .
\end{aligned}
$$

Equation [C8c] displays the explicit angular dependence of $m_{+1,0}^{( \pm)}(\alpha, \beta, \gamma)$ on the Euler angle $\alpha$.

The following considerations further show that $m_{+1,0}^{( \pm)}(\alpha, \beta, \gamma)$ is independent of the Euler angle $\gamma$. From its definition (Eq. [19]), this function depends on the polar angle between the laboratory and molecular $z$ axes, i.e., on the Euler angle $\beta$. As shown above, it also depends on the orientation of the laboratory $x$ axis with respect to the $z, \tilde{z}$ plane; this is specified by the Euler angle $\alpha$, which is the rotation required to bring the laboratory $x$ axis into the $z, \hat{z}$ plane. However, because of the assumed uniaxial symmetry in the zfs tensor, it does not depend on the choice of the $\hat{x}$ and $\hat{y}$ axes in the molecular coordinate frame, i.e., on the Euler angle $\gamma$. Thus we can write

$$
m_{+1,0}^{( \pm)}(\alpha, \beta, \gamma)=e^{i \alpha} m_{+1,0}^{( \pm)}(0, \beta, 0)
$$

and, by a straightforward extension of this reasoning,

$$
m_{q, q^{\prime}}^{( \pm)}(\alpha, \beta, \gamma)=e^{i\left(q+q^{\prime}\right) \alpha} m_{q, q^{\prime}}^{( \pm)}(0, \beta, 0)
$$

To calculate the spatial average of $\tilde{\Theta}_{1}(\alpha, \beta, \gamma)$, the angular dependence of product functions of the form $D_{0, q}^{(j)}(\alpha, \beta, \gamma) m_{r, s}^{( \pm)}(\alpha, \beta, \gamma)$ is required. From Eqs. [C1] and [C9], any product of this form can be written

$$
\mathcal{D}_{0, q}^{(j)}(\alpha, \beta, \gamma) m_{r, s}^{( \pm)}(\alpha, \beta, \gamma)=e^{i(q+r+s) \alpha} \mathscr{D}_{0, q}^{(i)}(0, \beta, 0) m_{r, s}^{( \pm)}(0, \beta, 0)
$$

When $q+r+s=0$, as occurs in each term of Eq. [40], this product is independent of the Euler angles $\alpha$ and $\gamma$. Thus the spatial average of $\tilde{\Theta}_{1}(\beta)$ can be calculated simply from integration over the space of the polar angle $\beta$,

$$
\left(T_{1 p}\right)_{\mathrm{dip}}^{-1}=(-1)\left(\frac{3^{-1 / 2} \kappa}{r^{3}}\right)^{2} 2^{-1} \int_{0}^{\pi} \tilde{\Theta}_{1}(\beta) \sin (\beta) d \beta .
$$

\section{APPENDIX D}

\section{Trigonometric Forms of Selected Wigner Rotation-Matrix Elements}

The forms given here were evaluated from the tables of Buckmaster $(28,29)$. It should be noted that his conventions differ from those used here [which follow Ref. (23)], in that $\mathcal{D}_{q_{1}, \psi_{2}}^{(j)}$ in his tables correspond to $\mathcal{D}_{q_{2}, y_{1}}^{(j)}$ in our notation. His formulas, which are tabulated in terms of trigonometric functions of half angles, were converted to forms involving full angles using the following standard trigonometric identities:

$$
\begin{gathered}
\cos ^{2}(\beta / 2)=[1+\cos (\beta)] / 2 ; \\
\sin ^{2}(\beta / 2)=[1-\cos (\beta)] / 2 ; \\
\sin (\beta / 2) \cos (\beta / 2)=2^{-1} \sin (\beta) ; \\
\cos ^{2}(\beta / 2)-\sin ^{2}(\beta / 2)=\cos (\beta)
\end{gathered}
$$




\begin{tabular}{|c|c|c|c|}
\hline$j$ & $q_{1}$ & $q_{2}$ & $\mathcal{D}_{q_{1}, q_{2}}^{(j)}(\alpha, \beta, \gamma)$ \\
\hline 0 & 0 & 0 & 1 \\
\hline 1 & 0 & 0 & $\cos (\beta)$ \\
\hline 1 & +1 & +1 & $2^{-1}[1+\cos (\beta)] \exp [i(\gamma+\alpha)]$ \\
\hline 1 & -1 & -1 & $2^{-1}[1+\cos (\beta)] \exp [i(-\gamma-\alpha)]$ \\
\hline 1 & \pm 1 & 0 & $2^{(-1 / 2)} \sin (\beta) \exp [ \pm i \gamma]$ \\
\hline 1 & 0 & \pm 1 & $2^{(-1 / 2)} \sin (\beta) \exp [ \pm i \alpha]$ \\
\hline 1 & +1 & -1 & $2^{-1}[1-\cos (\beta)]$ \\
\hline 2 & 0 & 0 & $2^{-1}\left[3 \cos ^{2}(\beta)-1\right]$ \\
\hline 2 & 0 & \pm 1 & $\mp 2^{-1} 6^{1 / 2} \sin (\beta) \cos (\beta) \exp [ \pm i \alpha]$ \\
\hline 2 & \pm 1 & 0 & $\pm 2^{-1} 6^{1 / 2} \sin (\beta) \cos (\beta) \exp [ \pm i \gamma]$ \\
\hline 2 & \pm 1 & $\mp 1$ & $2^{-1}[1-\cos (\beta)][1+2 \cos (\beta)] \exp [\mp i \alpha] \exp [ \pm i \gamma]$ \\
\hline 2 & \pm 2 & 0 & $2^{-2} 6^{1 / 2} \sin ^{2}(\beta) \exp [ \pm 2 i \gamma]$ \\
\hline 2 & 0 & \pm 2 & $2^{-2} 6^{1 / 2} \sin ^{2}(\beta) \exp [ \pm 2 i \alpha]$ \\
\hline 2 & \pm 2 & \pm 2 & $2^{-2}[1+\cos (\beta)]^{2} \exp [ \pm 2 i \gamma] \exp [ \pm 2 i \alpha]$ \\
\hline 2 & \pm 2 & $\mp 2$ & $2^{-2}[1-\cos (\beta)]^{2} \exp [ \pm 2 i \gamma] \exp [\mp 2 i \alpha]$ \\
\hline 2 & \pm 2 & \pm 1 & $\pm 2^{-1}[1+\cos (\beta)] \sin (\beta) \exp [ \pm 2 i \gamma] \exp [ \pm i \alpha]$ \\
\hline 2 & \pm 1 & \pm 2 & $\mp 2^{-1}[1+\cos (\beta)] \sin (\beta) \exp [ \pm i \gamma] \exp [2 i \alpha]$ \\
\hline 2 & \pm 2 & $\mp 1$ & $\pm 2^{-1}[1-\cos (\beta)] \sin (\beta) \exp [ \pm 2 i \gamma] \exp [\mp i \alpha]$ \\
\hline 2 & \pm 1 & $\mp 2$ & $\pm 2^{-1}[1-\cos (\beta)] \sin (\beta) \exp [ \pm i \gamma] \exp [\mp 2 i \alpha]$ \\
\hline 4 & 0 & 0 & $\left(\frac{3}{8}\right)-\left(\frac{15}{4}\right) \cos ^{2}(\theta)+\left(\frac{35}{8}\right) \cos ^{4}(\theta)$ \\
\hline & & & $2^{-3} \times 10^{1 / 2} \sin ^{2}(\beta) \times\left\{3[1+\cos (\beta)]^{2}-8 \sin ^{2}(\beta)\right.$ \\
\hline 4 & \pm 2 & 0 & $\left.+3[1-\cos (\beta)]^{2}\right\} \times \exp [ \pm 2 i \gamma]$ \\
\hline T & 0 & \pm 2 & $\left.+3\left[1-\cos (\beta)^{2}\right]\right\} \times \exp [ \pm 2 i \alpha]$ \\
\hline
\end{tabular}

\section{ACKNOWLEDGMENT}

This work was supported by the U.S. Department of Agriculture in the form of Research Grant C/89CRCR-2344.

\section{REFERENCES}

I. I. SOLOMON, Phys. Rev. 99, 559 (1955).

2. N. Bloembergen, J. Chem. Phys. 27, 575 (1957).

3. N. Bloembergen and L. O. Morgan, J. Chem. Phys. 34, 842 (1961).

4. R. A. DWEK, "Nuclear Magnetic Resonance (NMR) in Biochemistry," Clarendon Press, Oxford, 1973.

5. T. Bayburt and R. R. SharP, J. Chem. Phys. 92, 5892 (1990).

6. R. R. ShaRP, J. Chem. Phys. 93, 6921 (1990).

7. J. Freed, G. V. Bruno, and C. Polnaszek, J. Chem. Phys. 55, 5270 (1971).

8. L.-P. Hwang, Mol. Phys. 49, 1341 (1983).

9. T.-H. R. Chen, S.-J. Den, And L.-P. Hwang, Proc. Natl. Sci. Council (Taiwan) A 8, 224 (1984).

10. N. Benetis, J. Kowalewski, L. Nordenskiold, H. Wennerstrom, and P.-O. Westlund, Mol. Phys. 48, 329 (1983). 
11. N. Benetis, J. Kowalewski, L. Nordenskiold, H. Wennerstrom, AND P.-O. Westlund, Mol. Phys. 50, 515 (1983).

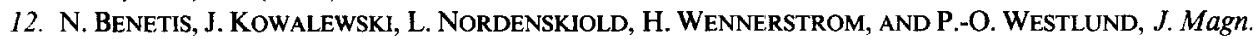
Reson. 58, 261 (1984).

13. P.-O. Westlund, H. WenNerstrom, L. Nordenskiold, J. Kowalewski, AND N. Benetis, J. Magn. Reson. 59, 91 (1984).

14. N. BENETIS AND J. KowALEWSKJ, J. Magn. Reson. 65, 13 (1985).

15. P.-O. Westlund, N. Benetis, And H. Wennerstrom, Mol. Phys. 61, 177 (1987).

16. S. SzYmanski, A. M. GryfF-Keller, AND G. BinsCH, J. Magn. Reson. 68, 399 (1986).

17. M. L. Cofield AND P. S. BRYAN, Inorg. Chim. Acta 112, I (1986).

18. B. J. KenNedY AND K. S. MURRAY, Inorg. Chem. 24, 1552 (1985).

19. B. J. KENNEDY AND K. S. MURRAY, Inorg. Chem. 24, 1557 (1985).

20. A. K. Greason, D. M. Doddrell, And P. Healy, Inorg. Chem. 17, 1216 (1978).

21. J. M. BOVET AND R. R. SHARP, unpublished data, 1990.

22. D. T. PegG, D. M. Doddrell, M. R. Bendall, and A. K. Gregson, Aust. J. Chem. 29, 1885 ( 1976 ).

23. B. L. SILvER, "Irreducible Tensor Methods," Academic Press, New York, 1976.

24. M. Rotenberg, R. Bivens, N. Metropolis, AND J. K. WoOten, JR., "The 3- $j$ and 6- $j$ Symbols," Technology Press, MIT, Cambridge, 1959.

25. A. AbraGaM, "The Principles of Nuclear Magnetism," Chap. VIII, Oxford Univ. Press, Oxford, 1961.

26. B. R. Stults, R. S. Marianelli, AND V. W. Day, Inorg. Chem. 18, 1853 (1959).

27. L.-P. Hwang and J. H. Freed, J. Chem. Phys. 63, 4017 (1975).

28. J. H. Freed, J. Chem. Phys. 68, 4035 (1978).

28. H. A. Buckmaster, Can. J. Phys. 42, 386 (1964).

29. H. A. Buckmaster, Can. J. Phys. 44, 2525 (1964).

30. S. Koenig, R. D. Brown, and J. Studebaker, Cold Spring Harbor Symp. Quant. Biol. 36, 551 (1971).

31. E. HADDY AND R. R. SHARP, Biochemistry 28, 3656 ( 1989 ). 